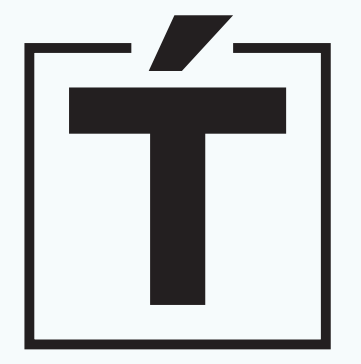

TEMPORÁNEA \#02 2021 


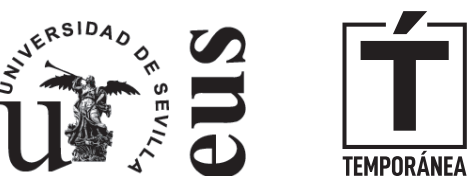




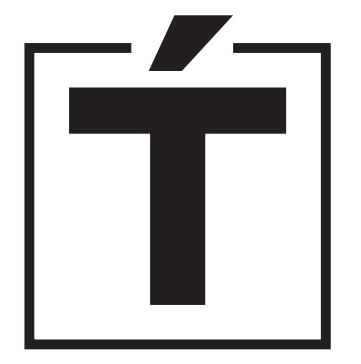

TEMPORÁNEA \#02 2021 


\section{T́ TEMPORÁNEA}

\#02 2021

\section{Directora}

Dra. Mar Loren-Méndez, Universidad de Sevilla, España

\section{Secretario}

Dr. Carlos Plaza, Universidad de Sevilla, España

\section{Coordinador de Redacción}

Dr. Daniel Pinzón-Ayala, Universidad de Sevilla, España

\section{Comité de Dirección}

Dra. Mar Loren-Méndez, Universidad de Sevilla, España

Dr. Carlos Plaza, Universidad de Sevilla, España

Dr. Daniel Pinzón-Ayala, Universidad de Sevilla, España

\section{Comité de Redacción}

Dra. María Carrascal, Universidad de Sevilla, España

Dr. Donetti Dario, University of Chicago, USA

Dra. Bianca De Divitiis, Università degli Studi di Napoli Federico II, Italia

Dra. María Elena Díez Jorge, Universidad de Granada, España

Dra. María Elia Gutiérrez Mozo, Universidad de Alicante, España

Dr. Carlos García Vázquez, Universidad de Sevilla, España

Dr. Fulvio Lenzo, Università IUAV di Venezia, Italia

Dra. Mar Loren-Méndez, Universidad de Sevilla, España

Dr. Emilio Luque, Universidad de Sevilla, España

\section{Comité Científico}

Dr. Fernando Agrasar Quiroga, Universidade da Coruña, España

Dra. Chiara Baglione, Politecnico di Milano, Italia

Dr. Gianluca Belli, Università degli Studi di Firenze, Italia

Dra. Cammy Brothers, Northeastern University, USA

Dr. Massimo Bulgarelli, Università IUAV di Venezia, Italia

Dr. Juan Calatrava Escobar, Universidad de Granada, España

Dra. Carolina B. García Estévez, Universitat Politècnica de Catalunya, España

Dr. Ignacio González-Varas Ibáñez, Universidad de Castilla-La Mancha, España

Dra. Maria Gravari Barbas, Université Paris 1 - Panthéon Sorbonne, France

Dr. Salvador Guerrero, Universidad Politécnica de Madrid, España

Dr. Fernando Marías, Universidad Autónoma de Madrid, España

Dra. Laura Martínez de Guereñu, IE University, España

\section{Diseño Gráfico}

Pedro García Agenjo, Coordinador

ISSN: 2695-7736

e-ISSN: 2659-8426

DOI: https://dx.doi.org/10.12795/TEMPORANEA

DEPÓSITO LEGAL: SE 32-2020

PERIOCIDAD DE LA REVISTA: Anual

IMPRIME: Digital Comunicaciones del Sur

EDITA: Editorial Universidad de Sevilla

LUGAR DE EDICIÓN: Sevilla

DIRECCIÓN CORRESPONDENCIA CIENTÍFICA:

E.T.S. de Arquitectura. Avda Reina Mercedes, 2, 41012, Sevilla

Mar Loren-Méndez, Dpto. Historia, Teoría y Composición Arquitectónicas

e-mail: temporanea@us.es

EDICIÓN ON-LINE:

Portal informático https://revistascientificas.us.es/index.php/temporanea Portal informático Editorial Univ. de Sevilla https://www.editorial.us.es/
Dra. Francesca Mattei, Università Roma Tre, Italia Dr. Daniel Pinzón-Ayala, Universidad de Sevilla, España Dra. Eleonora Pistis, Columbia University, USA Dr. Carlos Plaza, Universidad de Sevilla, España Dr. Pablo Rabasco, Universidad de Córdoba, España Dr.William Rey Ashfield, Universidad de la República, Uruguay Dr.Victoriano Sainz Gutiérrez, Universidad de Sevilla, España Dr. Julián Sobrino Simal, Universidad de Sevilla, España
(C) EDITORIAL UNIVERSIDAD DE SEVILLA, 2021

(C) TEXTOS: Sus autores, 2021

(C) IMÁGENES: Sus autores y/o instituciones, 2021 SUSCRIPCIONES, ADQUISICIONES Y CANJE: TEMPORÁNEA. Revista de Historia de la Arquitectura Editorial Universidad de Sevilla

Calle Porvenir, 27, 41013, Sevilla. Tel. 954487447 / 954487451 Fax 954487443 [eus4@us.es] [https://www.editorial.us.es]

Reservados todos los derechos. Ni la totalidad ni parte de esta revista puede reproducirse o transmitirse por ningún procedimiento electrónico o mecánico, incluyendo fotocopia, grabación magnética o cualquier almacenamiento de información y sistema de recuperación, sin permiso escrito de la Editorial Universidad de Sevilla.

Las opiniones y los criterios vertidos por los autores en los artículos firmados son responsabilidad exclusiva de los mismos. 


\section{Enfoque y alcance}

TEMPORÁNEA. Revista de Historia de la Arquitectura construye un foro internacional en el campo de la Historia de la Arquitectura. Colmando el vacío existente de publicaciones especializadas en esta materia en España, la revista tiene un marcado carácter internacional, que se traduce tanto en la participación activa de expertos internacionales en sus órganos como en las investigaciones que en ella se publican.

Se aborda la investigación en Historia de la Arquitectura desde cualquier disciplina, período cronológico y ámbito geográfico, y promueve la diversidad y complejidad de la Historia como valores irrenunciables. Junto con esta aproximación transversal y plural, esta publicación periódica defiende el carácter multiescalar de la arquitectura abarcando la historia del objeto construido, la ciudad y el territorio.

Se trata de una revista científica del sello Editorial de la Universidad de Sevilla EUS, que junto al equipo editorial de TEMPORÁNEA. Revista de Historia de la Arquitectura velará por la calidad, la transparencia y el rigor de la publicación. La revista va dirigida preferentemente a la comunidad científica y universitaria dedicada a la investigación en Historia de la Arquitectura y tendrá una periodicidad anual.

\section{Políticas de sección}

atemporánea se trata de una sección principal que aparecerá en todos los números. Dicha sección se compone de artículos de libre temática acordes con el perfil de la revista.

contemporánea se trata de una sección complementaria que aparecerá en todos los números. Dicha sección recogerá escritos de menor entidad tales como reseñas de exposiciones, recensiones de libros, entrevistas y en general temas de actualidad para la historia de la arquitectura.

extemporánea se trata de una tercera sección que aparecerá de manera eventual en determinados números de la revista. Dicha sección será de temática monográfica y estará compuesta por artículos.

\section{Proceso de evaluación por pares}

Tras el cierre del período de Llamada a Artículos / Call for articles, el Comité de Dirección evaluará la adecuación de las propuestas presentadas tanto a la temática y objetivos de la revista como a las normas establecidas para la redacción de los artículos. A continuación se procederá a la selección, con la ayuda de los comités de Redacción y Científico, de dos revisores/as de reconocido prestigio en la temática en cuestión para realizar una evaluación por el sistema de doble ciego. Los/as revisores/as realizarán sus consideraciones en base a los formularios de revisión en los formatos preestablecidos y en esta fase se garantizará el anonimato de autores/as y revisores/as. El artículo y los resultados de la evaluación por pares dobles ciegos se trasladarán al Comité de Redacción, que dictaminará, a la luz de los informes emitidos, qué trabajos serán publicados y, en su caso, cuáles precisarán de ser revisados y en qué términos. En caso de que los/as dos evaluadores/as aporten valoraciones opuestas, se procederá a solicitar una tercera evaluación.

Los resultados de la evaluación serán:

- Publicable: aceptado sin modificaciones.

- Requiere revisión: publicable con modifidaciones menores y sin necesidad de una segunda evaluación.

- Reevaluable: publicación con modificaciones mayores y precisa segunda evaluación.

- No publicable.

En el caso de que el artículo requiera modificaciones el/la autor/a recibirá los informes de los/as revisores/as. Junto con la nueva versión del artículo el/la autor/a deberá enviar una contestación justificada a dichos informes dirigido al Comité de Redacción. La nueva versión identificará aquellas modificaciones y será revisada por los/as mismos/as revisores/as.

TEMPORÁNEA. Revista de Historia de la Arquitectura publicará un número limitado de artículos por volumen y buscará el equilibrio entre las secciones, motivo por el cual, aunque un artículo sea aceptado o continúe en proceso de revisión, podrá quedar aplazado para ser publicado en un próximo número; en este caso, el/la autor/a podrá retirar el artículo o incluirlo en el banco de artículos de los próximos números. 


\section{Declaración ética sobre publicación y buenas prácticas}

TEMPORÁNEA. Revista de Historia de la Arquitectura participa de la edición en acceso abierto que promueve la Universidad de Sevilla a través del portal informático de la Editorial Universidad de Sevilla, velando por la máxima difusión e impacto y por la transmisión del conocimiento científico de calidad y riguroso. Se compromete así con la comunidad académica en garantizar la ética y calidad de los artículos publicados, tomando como referencia el Código de Conducta y Buenas Prácticas para editores de revistas científicas que define el Comité de Ética de Publicaciones (COPE).

Todas las partes implicadas en el proceso de edición se comprometen a conocer y acatar los principios de este código.

El Equipo Editorial se responsabiliza de la decisión de publicar o no en la revista los trabajos recibidos, atendiendo únicamente a razones científicas y no a cualesquiera otras cuestiones que pudieran resultar discriminatorias para el/la autor/a. Mantendrá actualizadas las directrices sobre las responsabilidades de los/as autores/as y las características de los trabajos enviados a la revista, así como el sistema de arbitraje seguido para la selección de los artículos y los criterios de evaluación que deberán aplicar los/as evaluadores/as externos/as. Se compromete a publicar las correcciones, aclaraciones, retracciones y disculpas necesarias en el caso de que sea preciso y a no utilizar los artículos recibidos para los trabajos de investigación propios sin el consentimiento de los/as autores/as. Garantizará la confidencialidad del proceso de evaluación: el anonimato de evaluadores/as y autores/as, el contenido que se evalúa, el informe emitido por los/as evaluadores/as y cualquier otra comunicación que se emita por los diferentes comités. Asimismo, mantendrá la máxima confidencialidad ante posibles aclaraciones, reclamaciones o quejas que un/a autor/a desee enviar a los comités de la revista o a los/as evaluadores/as del artículo. Se velará por el respeto e integridad de los trabajos ya publicados, motivo por el que se será especialmente estricto con el plagio y los textos que se identifiquen como plagios o con contenido fraudulento, procediéndose a su eliminación de la revista o a su no publicación. La revista actuará en estos casos con la mayor celeridad que le sea posible.

Los/as autores/as se harán responsables del contenido de sus envíos, comprometiéndose a informar al Comité de Dirección de la revista en caso de que detecten un error relevante en uno de sus artículos publicados, para que se introduzcan las correcciones oportunas. Asimismo, garantizarán que el artículo y los materiales asociados sean originales y que no infrinjan los derechos de autor de terceros. En caso de coautoría, tendrán que justificar que existe el consentimiento y consenso pleno de todos los/as autores/as afectados/as y que no ha sido presentado ni publicado con anterioridad por ninguno/a de ellos/as en otro medio de difusión.

Los/as evaluadores/as externos/as-revisores/as se comprometen a hacer una revisión objetiva, informada, crítica, constructiva, imparcial y respetuosa del artículo, basándose su aceptación o rechazo únicamente en cuestiones ligadas a la relevancia del trabajo, su originalidad, interés, cumplimiento de las normas de estilo y de contenido acordes con los criterios editoriales. Respetarán los plazos establecidos (comunicando su incumplimiento al Comité de Dirección con suficiente antelación) y evitarán compartir, difundir o utilizar la información de los textos evaluados sin el permiso correspondiente de la dirección y de los/as autores/as.

TEMPORÁNEA. Revista de Historia de la Arquitectura mantiene siempre abierta la recepción de artículos de las temáticas de interés de la revista. Los artículos entran en el proceso editorial a medida que son recibidos. Los/as autores/as consultarán la fecha concreta en cada convocatoria específica.

Los artículos enviados deben corresponder a las categorías universalmente aceptadas como producto de investigación, ser originales e inéditos y sus contenidos responder a criterios de precisión, claridad y rigor.

\section{Directrices previas al envío}

Todas las directices previas al envío vendrán descritas en el portal Web de la revista en el apartado que así lo indica. Para más facilidad podrá encontrarse siguiendo el siguiente enlace:

https://revistascientificas.us.es/index.php/TEMPORANEA/about/submissions\#onlineSubmissions

\section{Declaración de privacidad}

Los nombres y las direcciones de correo electrónico introducidos en esta revista se usarán exclusivamente para los fines establecidos en ella y no se proporcionarán a terceros o para su uso con otros fines. 


\section{Calidad editorial}

La Editorial Universidad de Sevilla cumple los criterios establecidos por la Comisión Nacional Evaluadora de la Actividad Investigadora para que lo publicado por el mismo sea reconocido como "de impacto" (Ministerio de Ciencia e Innovación, Resolución 18939 de 11 de noviembre de 2008 de la Presidencia de la CNEAI, Apéndice I, BOE n ${ }^{\circ}$ 282, de 22.11.08). La Editorial Universidad de Sevilla forma parte de la U.N.E. (Unión de Editoriales Universitarias Españolas) ajustándose al sistema de control de calidad que garantiza el prestigio e internacionalidad de sus publicaciones.

\section{Números I (2020) y II (2021)}

\section{Estadísticas}

Artículos recibidos: 17

Artículos aceptados: 8

Artículos rechazados: 9

Tasa de aceptación de originales: $47 \%$

Tiempo de demora: 156 dias

\section{Evaluadores/as}

María Isabel Alba Dorado, Universidad de Málaga (España)

Marilda Azulay Tapiero, Universidad Politècnica de València (España)

Maria Beltramini, Università degli Studi di Roma "Tor Vergata" (Italia)

Francesco Benelli, Alma Mater Studiorum-Università di Bologna (Italia)

Rachel Boyd, Columbia University (United States of America)

Joao Cabral, Universidade de Lisboa (Portugal)

Alfonso Guajardo-Fajardo Cruz, Universidad de Sevilla (España)

Ricardo Hernán Medrano, Universidade Presbiteriana Mackenzie (Brasil)

Jorge León Casero, Universidad de Zaragoza (España)

Mercedes Linares Gómez del Pulgar, Universidad de Sevilla (España)

Rafael López Guzmán, Universidad de Granada (España)

Esther Mayoral Campa, Universidad de Sevilla (España)
Rebeca Merino del Río, Universidad de Sevilla (España)

Rubén Muñoz, Universidad de Bío-Bío (Chile)

Marco R. Nobile, Università degli Studi di Palermo (Italia)

Francisco Ollero Lobato, Universidad Pablo de Olavide (España)

Manuel Parada López de Corselas, Universidad de Valladolid (España)

Susanna Pasquali, Università degli Studi di Roma "La Sapienza" (Italia)

Elena Paulino Montero, Universidad Complutense de Madrid (España)

José Peral López, Universidad de Sevilla (España)

José Pérez de Lama, Universidad de Sevilla (España)

Ramón Queiro, Universidad de Sevilla (España)

Francisco J. Rodríguez Barberán, Universidad de Sevilla (España)

Ferrán Ventura, Universidad de Málaga (España) 


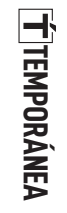

\begin{tabular}{rr} 
VI \\
\hline $02 \quad 2021$
\end{tabular} 


\section{atemporánea}

¿Os recibimos con alegría? Il viaggio di Olga Raggio a Vélez Blanco nel 1959: l'itinerario, gli interlocutori, i rapporti fra il Metropolitan Museum e le istituzioni spagnole

¿Os recibimos con alegría? El viaje de Olga Raggio a Vélez Blanco en 1959: el itinerario, los interlocutores, las relaciones entre el Metropolitan Museum y las autoridades españolas

Tommaso Mozzati.

La aljama cristianizada de Baeza en el siglo XIII, primera catedral de Andalucía

The Christianized Graet Mosque of Baeza in the 13th century, the oldest Andalusian Cathedral

Luis Rueda Galán

Sangallo, Vignola, Palladio and the Roman «Accademia de lo Studio de l'Architettura»

Sangallo, Vignola, Palladio y la «Accademia de lo Studio de l'Architettura» romana

Bernd Kulawik

Italo Calvino y Aldo Rossi en diálogo. Representaciones del monumento en la ciudad

Italo Calvino and Aldo Rossi in dialogue. Representations of the monument in the city Marc Fernández Cuyàs

\section{contemporánea}

Arquitectura de geometría variable

Architecture of variable geometry

Marta Pelegrín

Una nueva aportación a la historiografía arquitectónica de las Luces

A new contribution on Architectural Historiography of the Enlightenment

Juan Calatrava

El Plan General de Madrid de $\mathbf{1 9 8 5}$ y la reorientación del urbanismo tras la última burbuja inmobiliaria: de la expansividad insostenible a la regeneración urbana integrada

The 1985 Madrid General Plan and the reorientation of urbanism after the last real estate bubble: from unsustainable expansion to integrated urban regeneration

Pedro Górgolas. 
Editorial. Editorial. https://dx.doi.org/10.12795/TEMPORANEA.2021.02.08

Mar Loren-Méndez. https://orcid.org/0000-0002-1154-0526

Carlos Plaza. https://orcid.org/0000-0001-5632-2111

Daniel Pinzón-Ayala. https://orcid.org/0000-0002-2583-5077.

\section{atemporánea}

¿Os recibimos con alegría? Il viaggio di Olga Raggio a Vélez Blanco nel 1959: l'itinerario, gli interlocutori, i rapporti fra il Metropolitan Museum e le istituzioni spagnole. ¿Os recibimos con alegría? El viaje de Olga Raggio a Vélez Blanco en 1959: el itinerario, los interlocutores, las relaciones entre el Metropolitan Museum y las autoridades españolas. https://dx.doi.org/10.12795/TEMPORANEA.2021.02.01

Tommaso Mozzati. https://orcid.org/0000-0002-3204-6463.

La aljama cristianizada de Baeza en el siglo XIII, primera catedral de Andalucía. The Christianized Graet Mosque of Baeza in the 13th century, the oldest Andalusian Cathedral. https://dx.doi.org/10.12795/TEMPORANEA.2021.02.02

Luis Rueda Galán. https://orcid.org/0000-0001-9300-5764

Sangallo, Vignola, Palladio and the Roman «Accademia de lo Studio de l'Architettura». Sangallo, Vignola, Palladio y la «Accademia de lo Studio de l'Architettura» romana. https://dx.doi.org/10.12795/TEMPORANEA.2021.02.03 Bernd Kulawik. https://orcid.org/0000-0002-2083-6118

Italo Calvino y Aldo Rossi en diálogo. Representaciones del monumento en la ciudad. Italo Calvino and Aldo Rossi in dialogue. Representations of the monument in the city. https://dx.doi.org/10.12795/TEMPORANEA.2021.02.04 Marc Fernández Cuyàs. https://orcid.org/0000-0002-0080-9139

\section{contemporánea}

Arquitectura de geometría variable. Architecture of variable geometry. https://dx.doi.org/10.12795/TEMPORANEA.2021.02.05 Marta Pelegrín. https://orcid.org/0000-0003-0881-1169

Una nueva aportación a la historiografía arquitectónica de las Luces. A new contribution on Architectural Historiography of the Enlightenment. https://dx.doi.org/10.12795/TEMPORANEA.2021.02.06

Juan Calatrava. https://orcid.org/0000-0001-9401-9041.

El Plan General de Madrid de $\mathbf{1 9 8 5}$ y la reorientación del urbanismo tras la última burbuja inmobiliaria: de la expansividad insostenible a la regeneración urbana integrada. The 1985 Madrid General Plan and the reorientation of urbanism after the last real estate bubble: from unsustainable expansion to integrated urban regeneration.

https://dx.doi.org/10.12795/TEMPORANEA.2021.02.07

Pedro Górgolas. https://orcid.org/0000-0003-2178-875X 
atemporánea 


\section{Tommaso Mozzati}

https://orcid.org/0000-0002-3204-6463

tommaso.mozzati@unipg.it

Ricercatore presso il Dipartimento di Lettere all'Università degli Studi di Perugia, si è occupato in particolare di temi legati alla produzione scultorea italiana fra Quattro e Cinquecento. Ha pubblicato estensivamente sull'argomento, affrontando di volta in volta diverse prospettive di ricerca: dalla raffigurazione del nudo in epoca moderna alla fortuna di materiali come il marmo o il bronzo sul mercato artistico continentle, dalla presenza di maestranze toscane presso le princpali corti europee nel XVI secolo alla produzione di tombe per le monarchie transalpine.

Di recente, nel contesto di una borsa biennale presso l'ESDA Department del Metropolitan Museum of Art di New York, si è dedicato all'indagine delle vicende storiche e collezionistiche relative al patio di Vélez Blanco, presentando numerosi contributi al riguardo in convegni internazionali e in riviste specialistiche (dall'Archivo Español de Arte al Metropolitan Museum Journal). È stato borsista presso l'Italian Academy della Columbia University (New York), l'Harvard

Fecha de Recepción $24 \cdot$ Enero $\cdot 2020$ University Center for Italian Renaissance Studies (Fiesole), il Kunsthistorisches Institut in Florenz Max-Planck-Institut (Firenze).

Fecha de Aceptación $20 \cdot$ Julio $\cdot 2020$ 


\section{T́ TEMPORÁNEA}

\#02 2021

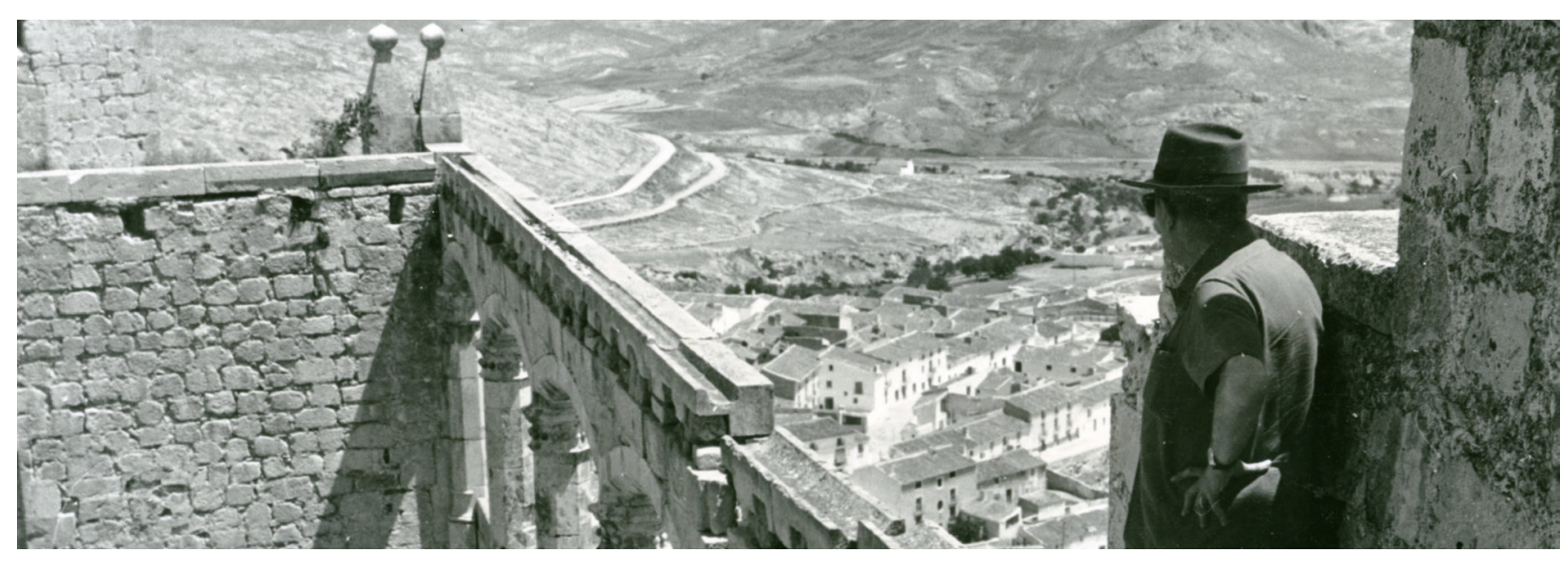

\section{¿Os recibimos con alegría? \\ Il viaggio di Olga Raggio a Vélez Blanco nel 1959: \\ l'itinerario, gli interlocutori, i rapporti fra il Metropolitan Museum e le istituzioni spagnole}

¿Os recibimos con alegría? El viaje de Olga Raggio a Vélez Blanco en 1959: el itinerario, los

interlocutores, las relaciones entre el Metropolitan Museum y las autoridades españolas

¿Os recibimos con alegría? Olga Raggio's trip to Vélez Blanco in 1959: the itinerary, the interlocutors, the relations between the Metropolitan Museum and the Spanish institutions

\section{Tommaso Mozzati}

Universitá degli Studi di Perugia

\section{Riassunto:}

L'articolo intende ripercorrere il dialogo apertosi fra il Metropolitan Museum of Art di New York, le autorità ministeriali spagnole e l'intelligentsia andalusa negli anni compresi fra il $1959 \mathrm{e}$ il 1963, cioè durante la ricostruzione del patio proveniente dal castello di Vélez Blanco nelle sale dell'istituzione newyorkese. Nel far questo il contributo si propone di ricollocare una simile rete di relazioni sullo sfondo della più ampia situazione politica e diplomatica vissuta in quegli anni dal regime franchista all'interno della comunità internazionale e, in particolare, nel contesto dei negoziati intrattenuti al tempo con il governo di Washington.

Parole chiavi: Vélez Blanco; patio; Metropolitan Museum of Art; franchismo; relazioni ibero-americane; museografia. 
El artículo traza la historia de las relaciones del Museo Metropolitano de Arte de Nueva York con las autoridades ministeriales españolas y algunos hombres de cultura activos en Andalucía entre 1959 y 1963, es decir, durante la reconstrucción del patio del castillo de Vélez Blanco en las salas del museo americano. Al hacerlo, la contribución también reubica este diálogo en el contexto de la más amplia situación política y diplomática experimentada por el régimen de Franco en esos años dentro de la comunidad internacional y, en particular, en el contexto de las negociaciones mantenidas en ese momento con el gobierno de Washington.

Palabras Clave: Vélez Blanco; patio; Museo Metropolitano de Arte; Franco; relaciones iberoamericanas; museografia.

Abstract:

The article analyzes the dialogue held between the Metropolitan Museum of Art, the Spanish ministerial authorities and the Andalusian cultural milieu between 1959 and 1963, around the reconstruction of the patio of Vélez Blanco in the main building of the New York institution. This contribution also aims to contextualize this three-sided relationship within a broader political and diplomatic context: in particular it focuses on the international position of the Franco regime and on the negotiations held at the time with the US government in Washington

Keywords: Vélez Blanco; patio; The Metropolitan Museum of Art; Franco; ibero-American relationship; museography. 
Spain I miss, of course, and dancing to flamenco music late at night

Ava Gardner, The Secret Conversations, 2013

Quando Olga Raggio giungeva in Spagna nella tarda primavera del 1959 come assistant curator del Metropolitan Museum di New York in missione per il Department of Renaissance and Modern Art, il suo arrivo si inseriva nel contesto di un clima diplomatico in progressivo miglioramento ${ }^{1}$.

Lungo gli anni Cinquanta, infatti, le relazioni fra gli Stati Uniti e la Spagna dominata dal Generalísimo Francisco Franco erano andate modificandosi verso una nuova stagione di rapporti che dissipava le ambiguità sorte nel 1945 in seguito alle conferenze di San Francisco e Potsdam².

In quell'anno (e a causa delle conclusioni raggiunte nei due summit internazionali), il paese era stato escluso dalla neonata Società delle Nazioni e un simile provvedimento si era allora costituito come la conseguenza diretta della vicinanza dimostrata dal Caudillo nei confronti delle potenze dell'Asse, uscite sconfitte dal secondo conflitto mondiale; del resto la Germania e l'Italia nazi-fasciste avevano attivamente sostenuto la sua vittoria negli anni tribolati della Guerra Civile, tra la sollevazione dei golpisti del 17 luglio

5 1936 e la caduta del governo democratico di Juan Negrín.

In seno a quegli incontri, gli Stati Uniti guidati da Harry S. Truman, in accordo con la Gran Bretagna di Winston Churchill, si erano però opposti ad azioni repressive contro la dittatura franchista, pur favorendone l'isolamento sullo scacchiere europeo: le pressioni russe in direzione di un interventismo nella politica interna spagnola erano state così arginate, distinguendo di fatto fra la condanna al regime e le sofferenze, i bisogni della popolazione civile.

Un anno di svolta, nel senso di un ristabilimento di legami diplomatici amichevoli, va individuato nel 1953. Nel settembre venne firmato il Patto di Madrid, un accordo secondo il quale gli Stati Uniti garantivano alla Spagna aiuti economici e militari, in cambio dell'utilizzo della rete nazionale di basi dell'esercito per le operazioni delle proprie forze armate; appena due anni dopo, e cioè nel 1955, l'azione concertata da Franco e dalla sua élite di governo per accreditarsi secondo coordinate e alleanze inedite nell'emisfero occidentale raggiunse gli obiettivi sperati, traducendosi in un accoglimento del paese in seno all'assemblea delle Nazioni Unite.

I miei ringraziamenti vanno a Denise Allen, Sarah Lawrence, José Domingo Lentisco Puche, James Moske, Luke Syson.

1 Notizia del viaggio è data per la prima volta in: RAGGIO, Olga. The Vélez Blanco patio: an Italian Renaissance Monument from Spain. In: The Metropolitan Museum of Art Bulletin. 1964, vol. 23, n. ${ }^{\circ}$ 4, p. 143. V. anche: RAGGIO, Olga. El patio de Vélez Blanco: un monumento señero del Renacimiento. GÓMEZ-MORENO, Carmen (trad.). In: Anales de la Universidad de Murcia Filosofía y Letras. 1968, vol. XXVI, n. os 2-3, p. 234.

2 V. ad esempio: MORADIELLOS, Enrique. The Potsdam Conference and the Spanish Question. In: Contemporary European History. 2001, vol. X, n. ${ }^{\circ}$ 1, pp. $73-90$ 


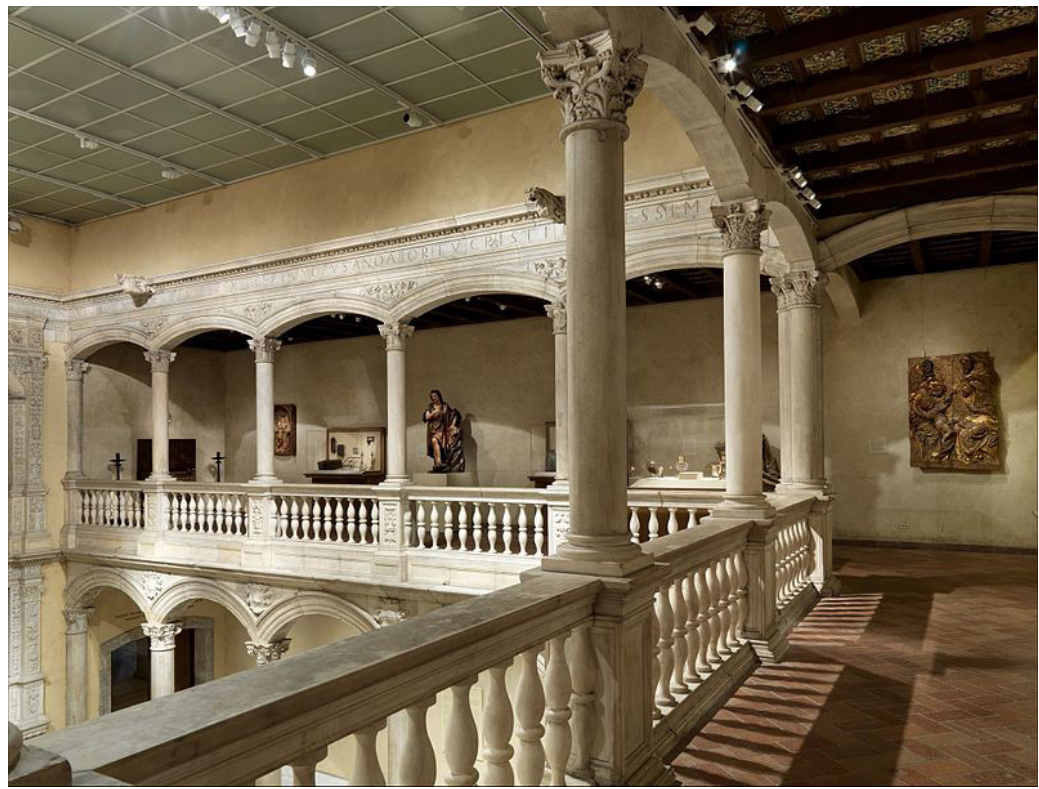

Figura 1. Patio di Vélez Blanco, ca. 1506/1509 - 1515.

Anche sul piano della vita civile, una politica siffatta ebbe conseguenze dirette di interscambio fra $i$ due paesi: tra $i$ fenomeni più ampiamente studiati, relativi a questo rinnovato clima di reciproca confidenza, è quello del turismo americano rivolto alla Spagna, crescente lungo il corso degli anni Cinquanta (e poi degli anni Sessanta) grazie alle strategie concertate da attori come il Ministerio de Información y Turismo e lo Spanish National Tourist Office ${ }^{3}$.

Un cambio di clima a tal punto radicale potrebbe spiegare, in qualche misura, la spedizione intrapresa dalla Raggio.

La curatrice aveva infatti deciso di recarsi oltreoceano per facilitare la risoluzione di una pratica annosa, il cui esito incerto stava occupando la direzione del Met dall'inizio del decennio precedente. Il fascicolo riguardava la donazione al museo da parte di George Blumenthal di una grandiosa struttura architettonica proveniente dal castello di Vélez Blanco, un cortile costituito da una doppia galleria porticata, comprensivo di finestre e soglie monumentali (fig. 1), commissionato all'alba del XVI secolo da Pedro Fajardo y Chacón quando la fortezza gli era stata assegnata da Isabella la Cattolica in cambio del controllo sulla città di Cartagena.
Il patio, venduto nel 1904 all'antiquario J. Goldberg e trasferito in tutta fretta a Parigi, rimase per qualche anno sull'ampia piazza del mercato d'arte internazionale, interessando eventuali acquirenti sull'una e l'altra sponda dell'Atlantico. Blumenthal lo comprò soltanto nel 1910, destinandolo alla spaziosa residenza che intendeva farsi costruire a Manhattan dalla firma Trowbridge \& Livingston, una palazzina in stile neorinascimentale su Park Avenue e la Settantesima edificata fra il 1911 e il 1912 per accogliere i marmi sottratti alla fortezza andalusa (fig. 2$)^{4}$.

Un dono tanto voluminoso era stato formalizzato dal lascito sottoscritto dallo stesso Blumenthal, attivatosi alla sua morte nel giugno 1941: assieme al patio, sarebbero giunti al museo numerosi, altri tesori delle collezioni del banchiere, che in seno al Met aveva rivestito il ruolo di presidente a partire dal 1934. In realtà, le sue intenzioni erano quelle di costituire la propria dimora nelle forme di una sede 'distaccata' della prestigiosa istituzione (certo sul modello dei Cloisters, il complesso espositivo inaugurato nel 1938 dalla famiglia Rockfeller nella parte settentrionale dell'isola di Manhattan, su suggerimento di Wilhelm R.Valentiner) ${ }^{5}$.

3 A questo proposito si può far riferimento all'utile résumé contenuto in ROSENDORF, Neal M. Franco sells Spain to America. Hollywood, Tourism and Public Relations as Postwar Spanish Soft Power. New York: Palgrave Macmillan, 2014, pp. 12-47.

4 LENTISCO PUCHE, José Domingo (coord.). El castillo de Vélez Blanco 1506-2006. Imagen y Memoria. Almería: Centro de Estudios Velezanos, 2007, pp. 90-121; MERINO DE CÁCERES, José Miguel; MARTÍNEZ RUIZ, María José. La destrucción del patrimonio artístico español. W. R. Hearst: el gran acaparador. Madrid: Cátedra, 2012, pp. 52-56. Per un'aggiornata ricostruzione di questa catena di eventi v.: MOZZATI, Tommaso. Storia collezionistica del patio di Vélez Blanco: nuovi documenti e fotografie inedite. In: BSAA Arte. 2019, n. ${ }^{\circ}$ 85, pp. 339-358; MOZZATI, Tommaso. La venta del patio de Vélez Blanco en 1904. In: Revista velezana. 2020, n. ${ }^{\circ} 38$, pp. 2-11; MOZZATI,Tommaso. El patio de Vélez Blanco y la residencia de George Blumenthal en New York. In: Revista velezana. 2020, n. ${ }^{\circ} 38$, pp. 13-23.

5 Sulla storia dei Cloisters: HUSBAND, Timothy B. Creating the Cloisters. In: The Metropolitan Museum of Art Bulletin. 2013, vol. 70, n. ${ }^{\circ} 4$, pp. 1, 4-48. 


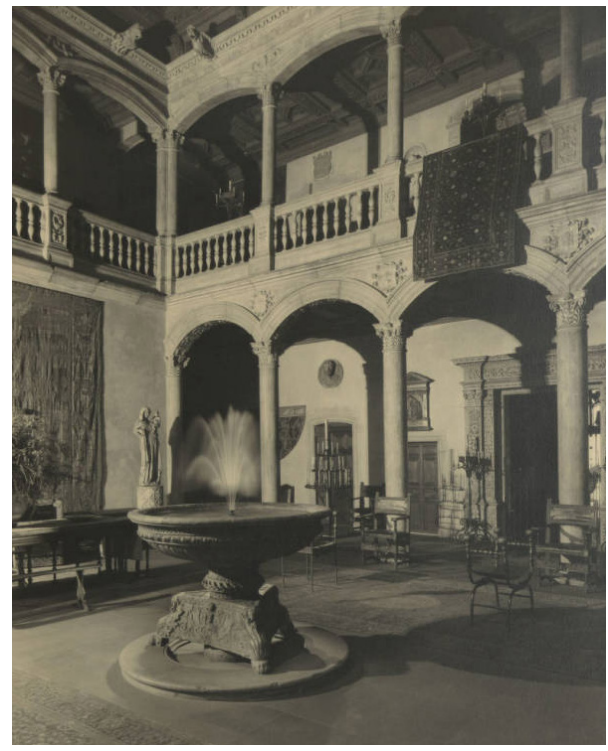

Figura

2. Mattie

Edwards

Hewitt,

Casa

Blumenthal,

1928.

\section{Figura 3. \\ Autore \\ sconosciuto, \\ Casa \\ Blumenthal, \\ 1941.}

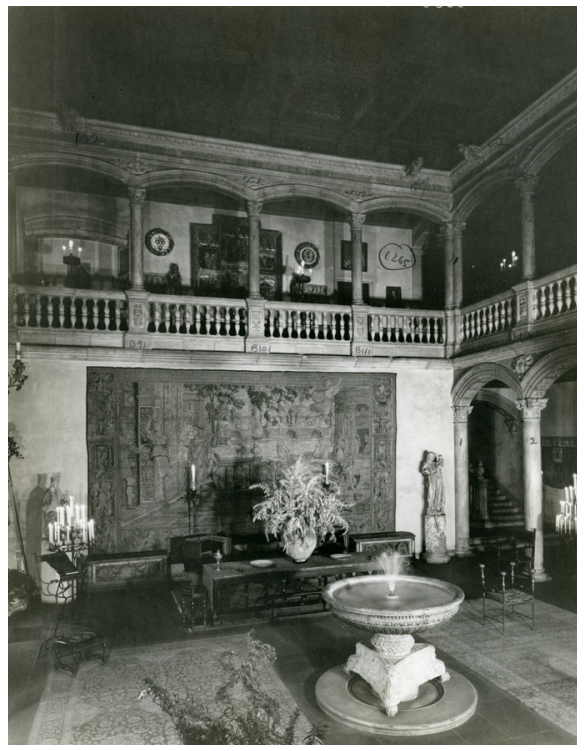

integrazioni commesse da Blumenthal e dai suoi architetti per adattare le forme del cortile alla sua destinazione newyorkese (fig. 3$)^{7}$.

D'altronde, l'inserimento del patio nella stratificata mappa del museo era apparsa possibile poiché il suo direttore, Francis H. Taylor (nominato nel 1940), stava immaginando un grandioso ampliamento degli spazi da finanziare attraverso un'estensiva raccolta fondi; un piano condannato a scontrarsi con le sue stesse mirabolanti ambizioni, vanificando di fatto l'intera campagna di riammodernamento programmata per gli ormai inappropriati ambienti dell'edificio sulla Quinta ${ }^{8}$. In un simile contesto, l'ingombro delle casse contenenti i marmi diVélez Blanco, trasferite nelluglio-agosto 1944 «in the AmericanWing courtyard (lower level) under a woodenroofed shed", si trasformò rapidamente in un problema pressante, da risolversi attraverso un'azione improcrastinabile? primis di Preston Remington)- rispetto alle

6 Per il testo del lascito v. New York, Metropolitan Museum of Art Archives, Francis H. Taylor Records, National Museum (Cuba), Secretary's File, 1948-53: memorandum al Board of Trustees, datato 1 novembre 1952. Dà prontamente conto della complessa gestione del lascito Blumenthal TAYLOR, Francis Henry. The Blumenthal Collection. In: The Metropolitan Museum of Art Bulletin. 1941, vol. 36, n. ${ }^{\circ}$ 10, pp. 193, 195-198. I contrasti di opinioni sorti al proposito sono riflessi anche in alcuni documenti conservati nell'archivio del Metropolitan: esemplare al riguardo è, ad esempio, uno scambio epistolare risalente al febbraio 1941 fra G. Lauder Greenway, secretary del museo, e Goodhue Livingston, partner della firma che si era occupata della costruzione della palazzina. Il primo infatti, scrivendo all'architetto, poteva affermare, l'11 del mese, come «the desire of Mr. Blumenthal as expressed in its will was for the Museum to demolish his residence after removing the architectural elements» [New York, Metropolitan Museum of Art Archives, Office of the Secretary. Correspondence Files 1870-1950. Blumenthal George. Bequest - Patio (and Pipe Organ) 1942-1946, 1948-1951:1ettera dell'11 febbraio, 1942 firmata G. Lauder Greenway a Goodhue Livingston]; l'architetto si sentiva invece titolato a rispondere: «I regret very much that the house is to be demolished as I know for many years it was Mr. Blumenthal's intention to retain it as a Branch of the Metropolitan Museum providing an endowment for that purpose" [New York, Metropolitan Museum of Art Archives, Office of the Secretary. Correspondence Files 1870-1950. Blumenthal George. Bequest - Patio (and Pipe Organ) 1942-1946, 1948-1951: lettera del 13 febbraio 1942 di Trowbridge \& Livingston Architects a G. Lauder Greenway].

7 Su queste pratiche si vedano i documenti conservati in New York, Metropolitan Museum of Art Archives, Office of the Secretary. Correspondence Files 1870-1950. Blumenthal George. Bequest - Patio (and Pipe Organ) 1942-1946, 1948-1951.

8 HECKSCHER, Morrison H.The Metropolitan Museum of Art. An architectural History. In: The Metropolitan Museum of Art. Bulletin. 1995, vol. 53, n. ${ }^{\circ} 1$, pp. $30-53$.

9 New York, Metropolitan Museum of Art Archives, Office of the Secretary. Correspondence Files 1870-1950. Blumenthal George. Bequest - Patio (and Pipe Organ) 1942-1946, 1948-1951. 


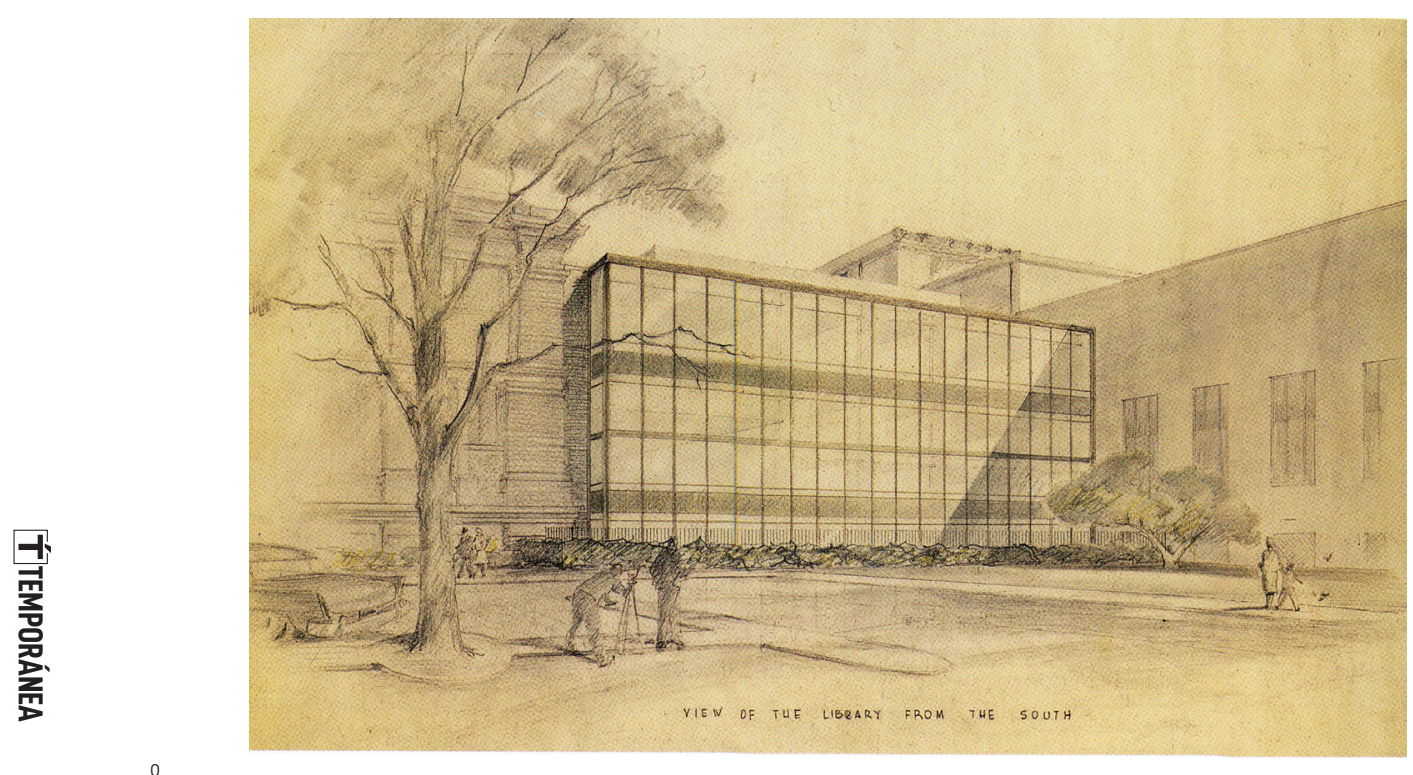

Figura 4. Brown,

Lawford and

Forbes, Veduta

meridionale della

Thomas J. Watson

Library, 1958.

\section{$\overline{\# 02 \quad 2021}$}

Per questo, in un torno d'anni brevissimo (compreso fra il 1949 e il 1952), si arrivò a formulare diverse ipotesi circa il futuro della struttura, immaginando perfino un suo invio a La Havana (per l'erigendo Museo de Bellas Artes) o a Puerto Rico (al Forte San Juan, al museo o all'università locali); mozioni tutte sostenute da Taylor ma avversate dalle resistenze del Board of Trustees, i cui membri a larga maggioranza si opposero al principio di scorporo dei beni già Blumenthal nel rispetto di una rigida lettura del suo testamento (senza contare che, nel '51, si era infine provveduto a trasportare le casse nei tunnel del Met, affrontando spese ingenti) ${ }^{10}$.

Un tanto rocambolesco susseguirsi di audaci iniziative e retromarce repentine si protrasse quindi fin dentro agli anni Cinquanta; solo nel 1954-1956 la progettazione di una nuova biblioteca all'interno del Met, su disegno dello studio Brown, Lawford \& Forbes, predispose infatti un'area adeguata per accogliere il cortile di Vélez Blanco (fig. 4$)^{11}$. È pertanto plausibile che, in quel momento specifico, la Raggio si appellasse al nuovo clima politico e culturale instauratosi fra gli Stati Uniti e la Spagna, mentre era impegnata a immaginare una ricostruzione filologicamente fedele del complesso; tanto più che in maniera significativa, fino alla prima metà del decennio, la diplomazia

intrattenuta per determinare la destinazione dei marmi non aveva incluso alcun referente spagnolo.

D'altra parte, il piano previsto dagli architetti poneva sfide sostanziali, relative proprio all'assetto del patio. Incluso in un'area di passaggio di forma regolare con funzione di ingresso scenografico per l'erigenda biblioteca, la sua organizzazione interna apriva a diversi interrogativi. Non si deve sottovalutare quanto la Raggio, scelta per rispondere ai quesiti storico-artistici sotto alla supervisione di James J. Rorimer (direttore del museo dal 1955), potesse contare su un'iconografia poverissima, utile a documentare l'originaria istallazione dei singoli elementi nel cuore della rocca di Vélez Blanco. Il corpus noto alla studiosa si limitava infatti a un acquerello prodotto al momento della vendita nel 1904 (fig. 5) e a poche altre foto, utilizzate dalla precedente bibliografia, per lo più risalente all'inizio del secolo XX; scatti parziali, non intesi per offrire un'immagine organica delle gallerie giunte in dono al Met. A tali testimonianze si aggiungeva la sola pianta della fortezza, inclusa nel primo volume dell'Arquitectura civil española di Vicente Lampérez y Romea, pubblicato a Madrid nel 1922: una planimetria che lo stesso autore dichiarava desunta da un modello 


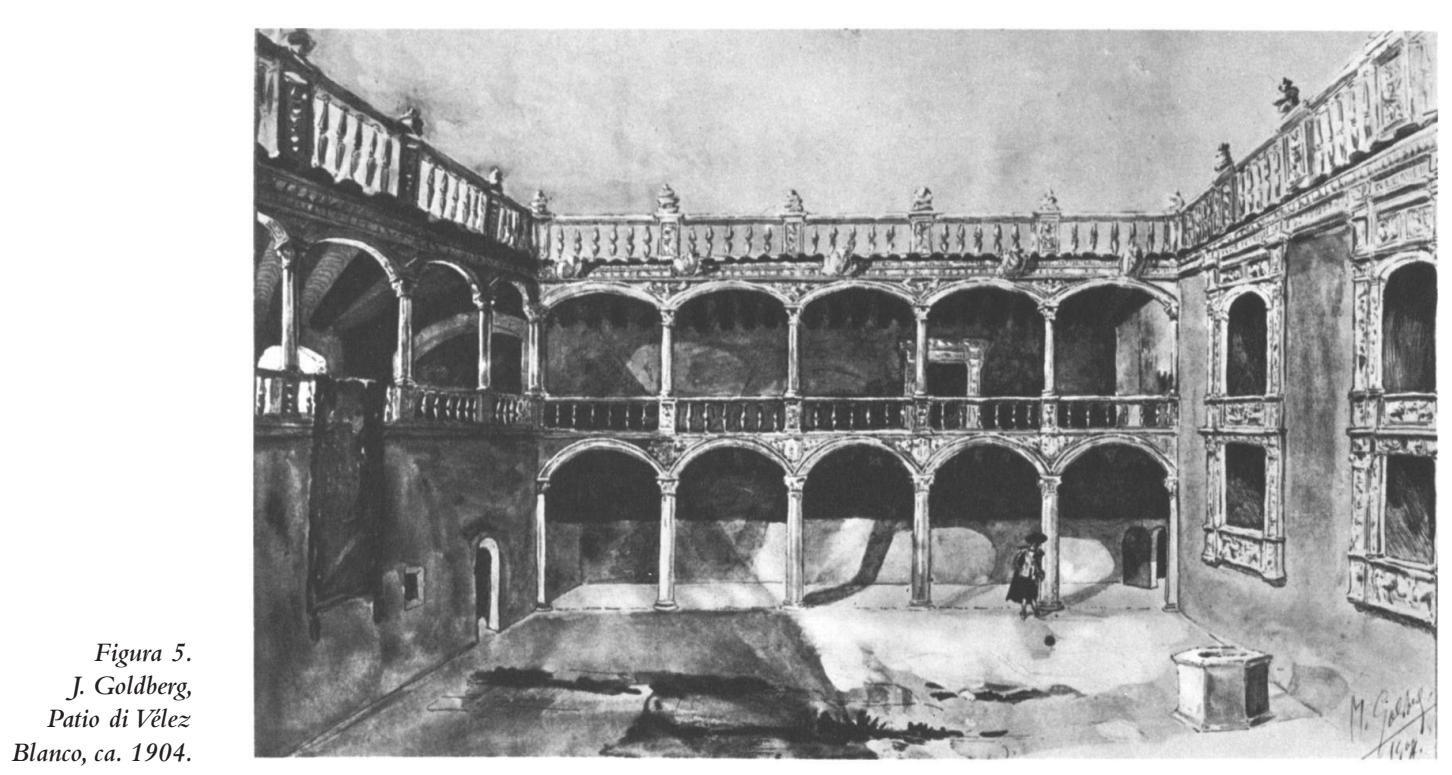

ligneo, al tempo nelle mani dei proprietari del complesso fortificato ${ }^{12}$.

Per questo motivo, le prime discussioni intercorse fra la Raggio e gli architetti dovettero piuttosto riferirsi ai materiali, maggiormente consistenti, relativi all'allestimento in casa Blumenthal. Lo attesta una corposa corrispondenza che documenta gli sforzi congiunti, volti a determinare la lunghezza delle logge, il numero degli archi da inserire in ciascun braccio oltre alla distribuzione degli arredi lapidei, a partire proprio dalla documentazione fotografica concernente il grande salone della palazzina su Park Avenue ${ }^{13}$. Il dibattito, in questo senso, poteva far riferimento a ben due serie di scatti, entrambe ispirate a uno scrupolo di completezza: la prima, eseguita da Mattie Edwards Hewitt nel 1928 (su richiesta dei coniugi Blumenthal), l'altra invece frutto di uno dei sopralluoghi compiuti dallo staff del museo nella residenza, dopo la morte del banchiere nel $1941^{14}$.
È significativo ad esempio notare come in questa fase si stesse discettando sulla scansione delle luci nella galleria superiore, galleria il cui svolgimento sarebbe stato ribaltato al Met per esigenze legate alla disposizione della sala intesa per accogliere il patio. Nel basarsi soltanto sul confronto fra la pianta di Lampérez y Romea e le scelte adottate dallo studio Trowbridge \& Livingston per Blumenthal si arrivò infatti a ridurre da tredici a undici il numero degli archi, secondo un computo metrico rispettoso delle dimensioni attribuite al cortile dalla mappa del 1922. Anche l'articolazione della cornice apicale, dell'epigrafe e dell'architrave fu un tema di ampio confronto fra $i$ progettisti e lo staff del museo: lo studio analitico delle 'foto Blumenthal' evidenziava infatti la probabile esistenza di elementi originali mancanti nel lotto giunto a New York, secondo un censimento verificabile sulle medesime misurazioni annotate dal volume dell' Arquitectura civil española ${ }^{15}$.

Le lambiccate riflessioni espresse nell'arco di questo contraddittorio e consegnate ai

12 Su queste fonti v. in ultimo: MOZZATI, Tommaso. Storia collezionistica. Op. cit. (n. 4), pp. 339-345. Una prima, importante raccolta dell'iconografia di Vélez Blanco è contenuta in: LENTISCO PUCHE, José Domingo (coord.). El castillo de Vélez. Op. cit. (n. 4), pp. 90-121. Per il volume di Vicente Lampérez y Romea v.: LAMPÉREZ Y ROMEA, Vicente. Arquitectura civil española: de los siglos I al XVIII. Madrid: Saturnino Calleja, 1922, vol. I, pp. 288-291. Per nuove fonti relative all'istallazione del patio nel castello di Vélez Blanco v.: MOZZATI, Tommaso. The patio of Vélez Blanco: a new Drawing and the Courtyard of the Fajardo Castle. In: Archivo español de arte. 2019, vol. XCII, n. ${ }^{\circ}$ 367, pp. 261-276.

13 New York, Metropolitan Museum of Art, European Sculpture and Decorative Arts Department, Curatorial Files, 41.190.482: Blumenthal - installation-photos/notes/correspondence.

14 Su queste due campagne fotografiche v.: MOZZATI, Tommaso. Storia collezionistica. Op. cit. (n. 4), pp. 356-358. L'articolo corregge ampiamente le conclusioni raggiunte in: HIETIKKO, Brad. Los relieves artísticos del patio del Castillo de Vélez Blanco en la casa de los Blumenthal (Nueva York) 1913. In: Revista velezana. 2017, n. ${ }^{\circ}$ 35, pp. 2-17.V. anche, più di recente: MOZZATI, Tommaso. El patio de Vélez Blanco. Op. cit. (n. 4), pp. 13-23. 15 New York, Metropolitan Museum of Art, European Sculpture and Decorative Arts Department, Curatorial Files, 41.190.482: Blumenthal installation-photos/notes/correspondence: memorandum di Raggio a J. Goldsmith Phillips, 14 febbraio 1958; memorandum di Raggio a James J. Rorimer, 26 febbraio 1958; lettera di Raggio a Geoffry N. Lawford, 28 febbraio 1958; memorandum di Raggio a Rorimer, 17 marzo 1958 ; memorandum di Raggio a Rorimer, 30 marzo 1958; memorandum di Raggio a Rorimer, 22 luglio 1958; lettera di Raggio a Lawford, 4 agosto 1958; memorandum di Raggio a Rorimer, 16 gennaio 1959; memorandum di Raggio senza destinatario, 21 gennaio 1959; memorandum di Raggio a Rorimer, 23 gennaio 1959 


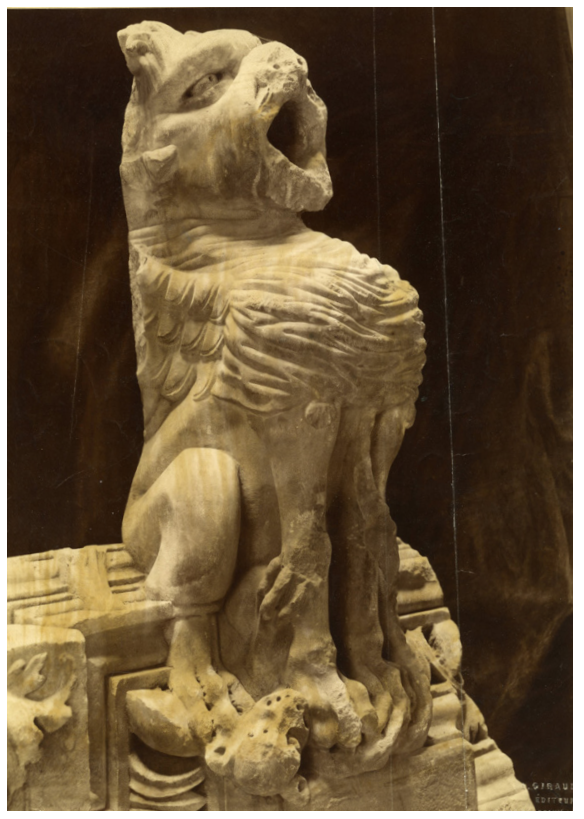

\#02 2021 messaggi informativi rivolti a Rorimer fra l'inverno e l'estate del 1958 bastano a giustificare la necessità, presto avvertita dalla Raggio, di raccogliere direttamente in Spagna ulteriori informazioni sul cortile, svolgendovi ricerche relative alla sua storia e all'organizzazione interna, con l'intento di riproporne l'architettura, nell'allestimento museale, in una chiave quanto più fedele all'impianto di partenza.

Una spedizione venne dunque prontamente calendarizzata, accorpandola a un ampio periplo europeo che si offre come un indice delle ricerche allora seguite dalla studiosa in relazione con il suo incarico ${ }^{16}$. Il viaggio si sarebbe articolato fra l'Italia, la Francia e la Spagna dal 20 aprile al 10 luglio del 1959: alle prime visite a Roma e Caprarola (per condurre indagini sul Tavolo Farnese, appena entrato nella raccolte del Met), dovette seguire un soggiorno a Parigi e infine una lunga permanenza spagnola. Grazie al report indirizzato a Rorimer il 21 luglio per adempiere alla burocrazia interna del museo sappiamo infatti che oltre a Madrid, dove la studiosa visitò il Prado ma anche la galleria di Arturo Linares, la sua traversata avrebbe toccato "Barcelona, Gerona, [...], Toledo, Valladolid, Cordoba, Sevilla, Granada, Almería and Murcia», in un vero e proprio Grand Tour; percorso che appare come una 'ricognizione' di territori per lo più estranei (almeno fino ad allora) agli interessi di
Figura 6. Jadolphe Giraudon, Frammento del patio di Vélez Blanco, ca. 1904-1905.

ricerca della Raggio. Se infatti le tappe di Almería e Murcia, assieme a diverse soste andaluse, appaiono legate allo studio su Vélez Blanco, le altre si presentano come il frutto di una sapiente indicizzazione dei centri imperdibili in una mappatura nazionale, utili tutt'al più alla corretta contestualizzazione della vicenda del castello dei Fajardo ${ }^{17}$.

È curioso, ad esempio, sottolineare quanto la Raggio apprezzasse le scelte museografiche accolte "at the Museum of Spanish Decorative Arts in Madrid and in several recently arranged provincial Spanish Museums (Granada, Murcia, Valladolid)», nel contesto di un itinerario durante il quale aveva ammirato anche gli allestimenti di Palazzo Bianco a Genova e del Museo Picasso ad Antibes. Tali notazioni aiutano a comprendere le finalità diversificate della sua trasferta, sollecitate a un tempo dalla curiosità intellettuale e dai bisogni variegati connessi alla posizione rivestita dalla studiosa all'interno del Met.

Più significativo delle mostre visitate su suolo iberico (Los castillos de Cataluña, organizzata a Barcellona dall'Asociación de los amigos de los Castillos) è poi l'elenco degli archivi fotografici con cui la Raggio prese contatto: quello dell'Instituto Amatller e dell'Archivo Mas, ma anche quelli del paludatissimo Instituto Velázquez di Madrid e del Laboratorio de Arte dell'Università 


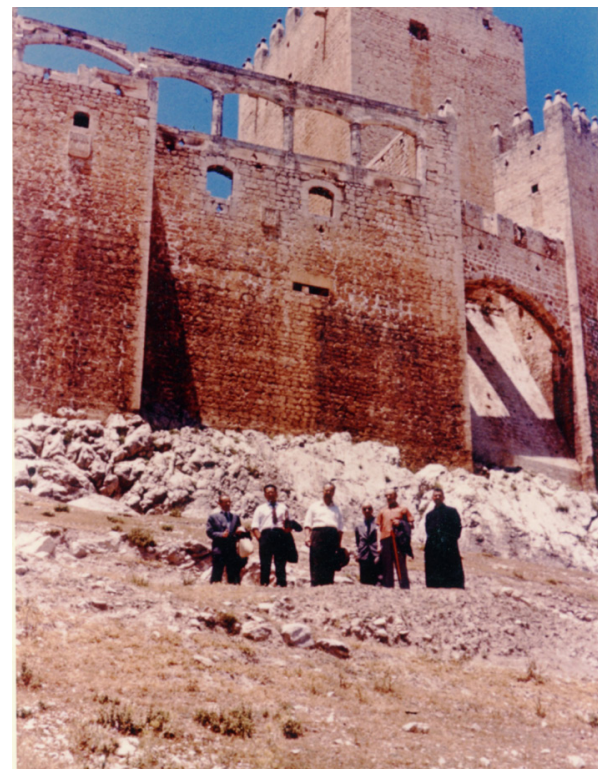

Figura 7. Olga Raggio, Castello di Vélez Blanco, 1959.

di Siviglia. A queste istituzioni la Raggio avrebbe inoltrato ordini consistenti (in totale oltre 180 immagini) da destinare «to the Museum's study collection»; del resto la stessa Raggio eseguì una serie di scatti in Kodacolor, ancora conservati nei files dell'European Sculpture and Decorative Arts Department. Una simile raccolta si riferiva particolarmente alla ricerca in corso sul patio di Vélez Blanco: d'altronde sappiamo da una sua nota autografa che già a Parigi la Raggio aveva seguito le tracce delle foto realizzate da Adolphe Giraudon attorno al 19041905 per commercializzare l'immagine dei frammenti marmorei, messi in vendita da Goldberg (fig. 6) ${ }^{18}$.

In un'agenda tanto ingombra di visite $\mathrm{e}$ incontri, il sopralluogo alla fortezza andalusa venne fissato per il 25 e il 26 giugno; tuttavia, l'approdo in una regione così isolata era stato preparato con molta cura, ricorrendo per l'appunto alla rete di relazioni che avevano accolto la studiosa al suo arrivo a Madrid.

L'aiuto più consistente le sarebbe arrivato da Francisco Prieto-Moreno, allora director general de Arquitectura e capo architetto de La Alhambra ${ }^{19}$. Per utilizzare le parole della studiosa, consegnate al suo report:

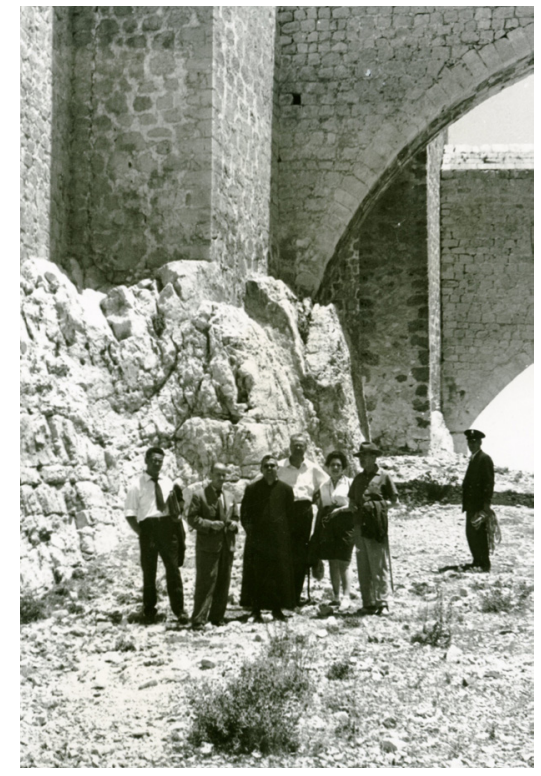

Figura 8. Francisco Prieto-Moreno, Castello di Vélez Blanco, 1959.

«As [the Castle of Vélez Blanco] is now abandoned and completely inaccessible without technical assistance, in order to visit it I had to arouse the interest and secure the cooperation of the State architect in charge of this part of Andalusia, Señor Don Francisco Prieto-Moreno y Pardo. [...] Mr. Prieto-Moreno organized an expedition to help me carry out my project. On June 2526 he drove me over there from Granada».

In un chiaro spirito di collaborazione fra istituti avrebbero preso parte alla spedizione anche Alfonso Gámir Sandoval, delegato regionale della Asociación de los amigos de los Castillos, e Jesús Bermúdez Pareja, direttore del Museo Arqueológico dell'Alhambra; sappiamo inoltre, da alcune più tarde testimonianze, che della comitiva dovettero far parte il sindaco diVélez Blanco, Miguel Ballesteros Motos, e José Ángel Tapia Garrido $^{20}$, già parroco nel pueblo andaluso (dal 1948 al 1955), autore proprio in quell'anno del libro di indagine storica Vélez Blanco, la villa señorial de los Fajardo ${ }^{21}$. Tutte queste presenze si ritrovano nelle fotografie scattate per l'occasione dalla Raggio e in quelle realizzate da Prieto-Moreno (figs. 7-8); documenti utili a rendicontare lo stato della fortezza alla fine del decennio, oltre che

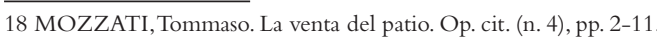

19 Su Prieto-Moreno v.: ROMERO GALLARDO, Aroa. Prieto-Moreno, arquitecto conservador de La Alhambra (1936-1978). Razón y sentimiento. Granada: Editorial Universidad de Granada, 2014, pp. 22-57.

20 GUIRAO GEA, Miguel. Se va a empezar a restaurar el castillo de Vélez Blanco. In: La voz de Almería. 19 agosto, 1964 , p. 6 (ripubblicato in: LENTISCO PUCHE, José Domingo (coord.). El castillo de Vélez Blanco. Op. cit. (n. 4), pp. 176-183).

21 TAPIA GARRIDO, José Ángel. Vélez-Blanco, villa señorial de los Fajardo. Vélez Blanco: Diputación Provincial De Almería, 1959. Su Tapia Garrido v. LENTISCO PUCHE, José Domingo (coord.). El castillo de Vélez Blanco. Op. cit. (n. 4), pp. 184-195. 


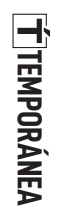

12

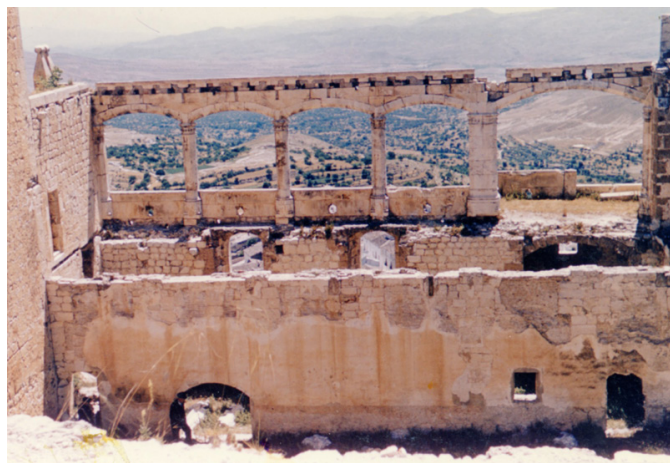

Figura 9. Olga Raggio, Castello di Vélez Blanco, 1959.

importanti per comprendere le osservazioni condotte in loco dalla studiosa.

La sua relazione ricorda infatti quanto il castello, alla prima ispezione, fosse «in relatively good state of preservation»; aggiunge inoltre che, sebbene «in many cases ceilings, floors, and staircases have collapsed, most of the outside and inside walls» si trovavano ancora intatti. L'area del patio risultava poi ampiamente leggibile, agevolando il riordino degli elementi marmorei posseduti dal Met (figs. 9-10).

Del resto, durante questa stessa permanenza spagnola, la Raggio si sarebbe interessata anche a rintracciare il modello ligneo cui aveva fatto ricorso Lampérez y Romea per il suo sondaggio sull'architettura civile. In un tal compito venne assistita da Gregorio Marañón, che si stava allora attivamente interessando al castello: nel 1960 sarebbe infatti apparso - postumo- il suo volume Los tres Vélez, prova dell'attenzione dello studioso per la struttura fortilizia e la sua storia ${ }^{22}$.

Il report chiarisce inoltre come, durante il sopralluogo, ci si fosse resi conti della presenza in paese e nella rocca di alcuni frammenti provenienti dal cortile, con ogni evidenza esclusi - per fretta o disattenzionedalla vendita del 1904 (fig. 11). La veridicità di una simile notizia è sostenuta da altre informazioni contenute nel resoconto: la

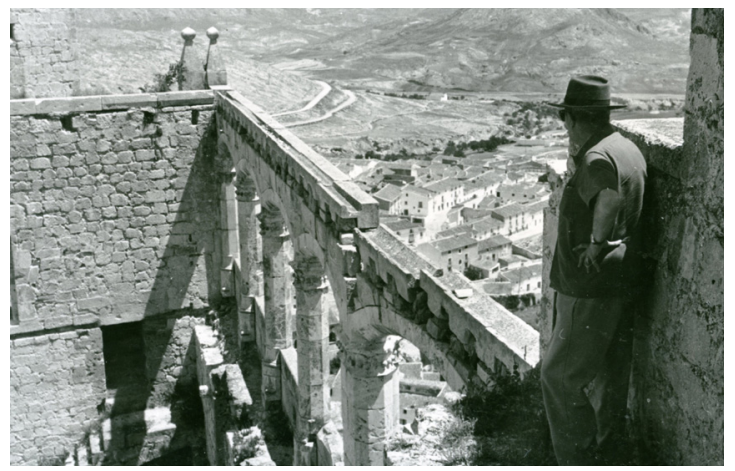

Figura 10. Francisco Prieto-Moreno, Castello di Vélez Blanco, 1959.

studiosa vi specifica che «neither PrietoMoreno nor the other officials who accompanied us had ever been there [before]». Un'eccezione, in questo senso, dovette esser rappresentata da Tapia Garrido (oltre che dal sindaco Ballesteros Motos) ${ }^{23}$, i quali poterono fungere pertanto da guida in una simile caccia al tesoro.

Si arrivò allora a identificare "on the floor of the Patio and down in the village of Vélez Blanco, outside and inside the main church of Santiago, [...] six or seven fragments of the egg-and-dart cornice», così come «the marble well that once stood in a corner [...] in a private house in Vélez Blanco».

Il documento riconduce al medesimo momento anche il primo pensiero relativo a un'eventuale acquisizione da parte del Met di questi materiali. La Raggio vi riferisce infatti di essersi al riguardo confrontata con Prieto-Moreno (e di aver poi anticipato la faccenda a Antonio Gallego Burín, director general de Bellas Artes) ${ }^{24}$, ottenendo in cambio risposte incoraggianti.

Si tratta ovviamente di asserzioni da leggersi in una prospettiva corretta. L'iniziale disponibilità raccolta dalla Raggio poté, almeno in qualche misura, esser motivata da una cortesia formale esercitata nei confronti della collega straniera, presentatasi come 'portavoce' di un'illustre istituzione culturale

22 MARAÑÓN, Gregorio. Los tres Vélez. Una historia de todos los tiempos. Madrid: Espasa-Calpe, 1960. Su Marañón durante gli anni successivi alla Guerra Civile v.: LÓPEZ VEGA, Antonio. Gregorio Marañón. Radiografía de un liberal. Madrid:Taurus, 2011, pp. 361-460.

23 Per un breve profilo biografico di Tapia Garrido v.: LENTISCO PUCHE, José Domingo (coord.). El castillo de Vélez Blanco. Op. cit. (n. 4), p. 184. 24 Sull' attività di Gallego Burín in quegli anni v.: GALLEGO MORELL, Antonio. Antonio Gallego Burín (1895-1961). Madrid:Tapa Blanca, 1973, pp. 115-127; VIÑES MILLET, Cristina. Antonio Gallego Burín. Granada: Comares, 2003, pp. 135-156. 


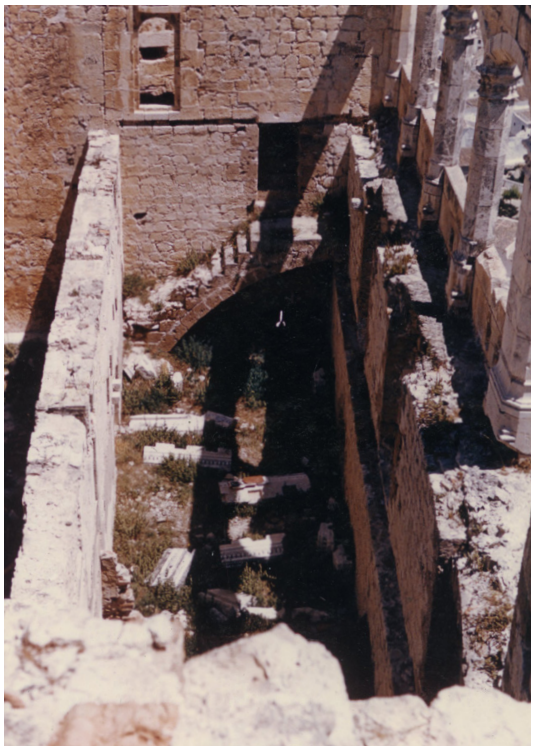

americana; e d'altronde la stessa Raggio, nel rendicontare il proprio soggiorno al direttore Rorimer, dovette preoccuparsi di mettere in evidenza i risultati più tangibili della spedizione, magari peccando d'ottimismo sull'effettiva disponibilità dimostratale dagli spagnoli.

È pur vero, tuttavia, che la susseguente corrispondenza intrattenuta da New York con Prieto-Moreno ne conferma la buona disposizione ${ }^{25}$.

Già durante il sopralluogo il funzionario aveva promesso di far seguire, al rientro della collega negli U.S.A., l'invio di una pianta dell'edificio, conservata fra $i$ documenti del Patrimonio Artístico, oltre che di materiale fotografico aggiuntivo centrato sulla fortezza e sulle sue pertinenze. In una lettera del 2 settembre, la Raggio gli avrebbe quindi rammentato l'impegno, approfittando dell'occasione per tornare su «another important question» e cioè «that of the fragments of the marble egg-anddart cornice of the Patio». Al proposito il messaggio ripeteva la volontà del Met, qualora si fosse concordato «a reasonable price", di procedere con l'acquisto di tutti gli elementi marmorei ancora nel castello, di quelli «on the street outside the door of the church of Santiago», oltre della coppia di marmi impiegati nella stessa chiesa per sorreggere «the Communion bench». A questa sollecitazione Prieto-Moreno avrebbe replicato, due settimane più tardi, offrendosi di condurre lui stesso una prima trattativa coi legittimi proprietari; servizio per il quale la Raggio lo avrebbe ringraziato il 22 settembre.

Ancora in novembre, il Met ricevette novità dalla Spagna, sebbene il loro tenore non fosse del tutto positivo. La lettera di PrietoMoreno, datata al 23, pur non rinnovando alcuna promessa, sottolineava infatti lo scarso rilievo sul piano istituzionale dell'eventuale acquisto; aggiungeva però come, durante una conversazione con Gallego Burín, gli fosse sembrato di vedere delinearsi all'orizzonte certi ostacoli di natura squisitamente politica ${ }^{26}$.

Nonostante la persistente cautela esercitata dallo spagnolo, la Raggio doveva tuttavia riporre più di una speranza nel buon esito della pratica. Lo dimostra una nota tachigrafica, consegnata a Rorimer sulla metà dell'autunno, infusa di ironia e incoraggiante ottimismo: «things are progressing, but... in a Mediterranean way» ${ }^{27}$.

25 Per la corrispondenza citata di seguito nel corpo dell'articolo v. New York, Metropolitan Museum of Art, European Sculpture and Decorative Arts Department, Curatorial Files, 41.190.482: Blumenthal - installation-photos/notes/correspondence.

26 La planimetria e le foto promesse da Prieto-Moreno arrivarono a New York solo dopo il febbraio 1960 (e prima del mese di maggio): v. New York, Metropolitan Museum of Art, European Sculpture and Decorative Arts Department, Curatorial Files, 41.190.482: Blumenthal - installation-photos/ notes/correspondence: lettera di Prieto-Moreno a Raggio, 8 febbraio 1960; memorandum di Arthur F. Klein (Supervisor for Plans and Construction), 25 maggio 1960; lettera di Raggio a Lawford, 24 giugno 1960.

27 New York, Metropolitan Museum of Art, European Sculpture and Decorative Arts Department, Curatorial Files, 41.190.482: Blumenthal installation-photos/notes/correspondence: memorandum di Raggio a Rorimer, 2 novembre 1960. 


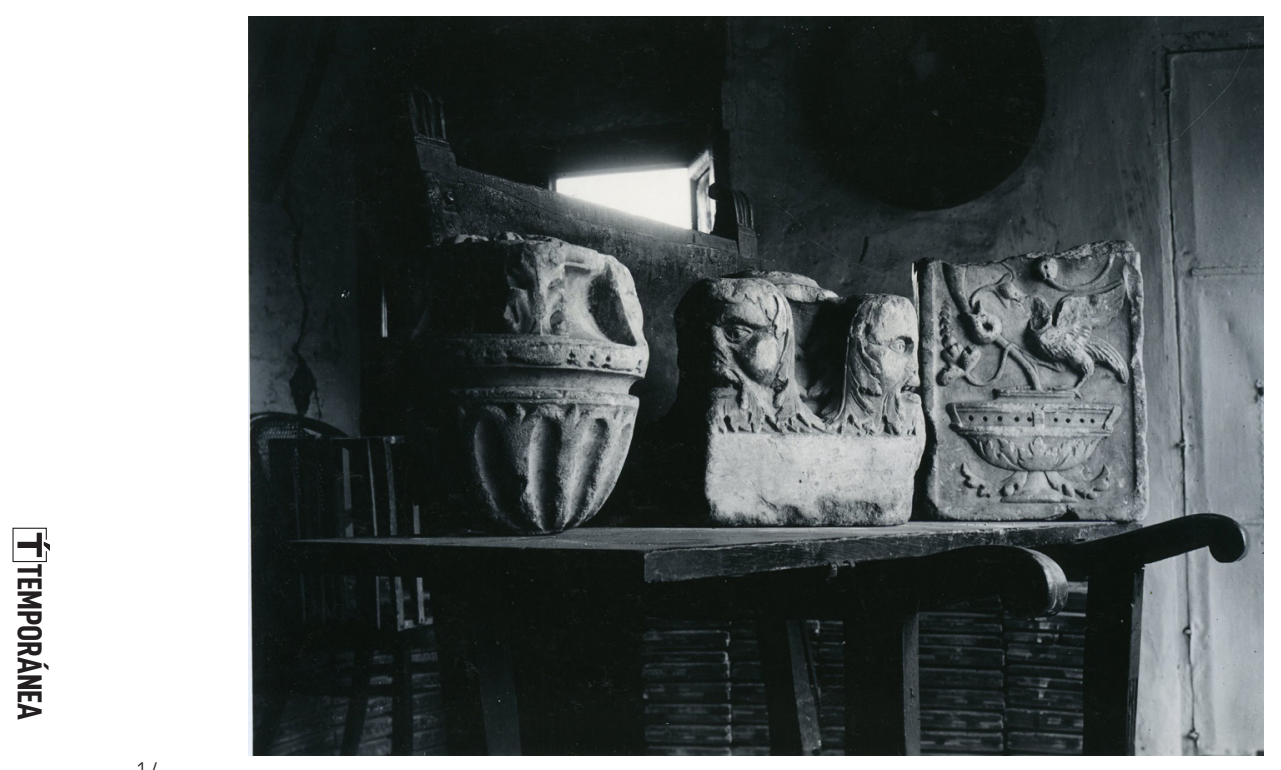

Figura 12. Leopoldo

Torres Balbás, Castello di

Vélez Blanco, ca. 1926.

Proprio la documentazione, prodotta all'interno del Metropolitan, consente del resto di valutare il durevole effetto dei rapporti stretti dalla studiosa con la Spagna.

Nel giugno seguente,la Raggio avrebbe infatti compilato per Rorimer un promemoria dal titolo «People to be contacted to obtain the pieces of marble egg-and-dart cornice that we need for the patio» ${ }^{28}$, elenco stilato con il contributo dei suoi corrispondenti, fra cui Leopoldo Torres Balbás, occupatosi negli anni Venti-Trenta del castello di Vélez nello svolgimento del suo ruolo di arquitecto restaurador della $6^{\text {a }}$ Zona $^{29}$.

La lista si apriva ovviamente col nome di Prieto-Moreno; di seguito, l'appunto menzionava i Marchesi di Valverde, oltre al vescovo di Almería, Alfonso Ródenas García. Se infatti i primi erano all'epoca i legittimi proprietari del castello, il secondo sembrava il referente più adatto per assicurarsi i frammenti della chiesa di Santiago. Tali

informazioni erano in parte state fornite alla Raggio da un'altra storica dell'arte, María Luisa Caturla (al tempo considerata fra le massime esperte dell'opera di Zurbarán), figura che -passati pochi mesi- avrebbe svolto un ruolo attivo nella vicenda del patio.

Risale infatti alla primavera/estate del 1961 un suo breve scambio epistolare con Rorimer, legato alla spinosa faccenda e con tutta evidenza- suscitato dalla nomina in Spagna del nuovo director general de Bellas Artes, Gratiniano Nieto Gallo. Il 5 giugno la Caturla informava infatti il suo corrispondente americano di essersi intrattenuta a pranzo con il funzionario neoeletto e che questi l'aveva autorizzata a comunicargli la disponibilità istituzionale alla cessione degli elementi del patio, in cambio però della reja della cattedrale di Valladolid (città cara a di Nieto), donata al Met nel $1956^{30}$. Non ricevendo risposta, la studiosa avrebbe riscritto il 12 luglio. Sicura che la

28 New York, Metropolitan Museum of Art, European Sculpture and Decorative Arts Department, Curatorial Files, 41.190.482: Blumenthal installation-photos/notes/correspondence: memorandum di Raggio a Rorimer, 2 giugno 1960.

29 Su Torres Balbás v.: GALlEGO ROCA, Francisco Javier (ed.). Epistolario de Leopoldo Torres Balbás a Antonio Gallego Burín. Granada: Editorial Universidad de Granada, 1995, pp. 18-23; MUÑOZ COSME, Alfonso. La vida y la obra de Leopoldo Torres Balbás. Sevilla: Junta de Andalucía, 2005; MOSQUERA ADELL, Eduardo. Torres Balbás y Andalucía. In: RUIZ CABRERO, Gabriel; MUÑ COSME, Alfonso; SÁNCHEZ DEL BARRIO, Antonio (ed.). Leopoldo Torres Balbás y la restauración científica. Sevilla: Instituto Andaluz del Patrimonio Histórico-Patronato de la Alhambra y el Generalife, 2013,pp. 129-156. A questo momento della carriera di Torres Balbás devono risalire le foto e le piante manoscritte del castello e delle sue pertinenze, conservate presso l'Archivo del Patronato de la Alhambra y Generalife (nn. F-01286-89; P-008546, P-008548, P-008554), databili attorno al 1926; su queste foto v.: LENTISCO PUCHE, José Domingo (coord.). El castillo de Vélez. Op cit. (n. 4), pp. 156-159. Alcune fotografie di Vélez Blanco, attribuite a Torres Balbás, sono conservate in copia anche presso i Curatorial Files del Met; stando agli scambi epistolari di Prieto-Moreno con la Raggio, dovettero essere inviate dal primo alla seconda, interessata a raccogliere quanta più documentazione possibile sul patio [fig. 12]; v. n. 26. Fra questi scatti, è inclusa un'immagine già pubblicata in: LENTISCO PUCHE, José Domingo (coord.). El castillo de Vélez. Op. cit. (n. 4), p. 115 (prima in alto a sinistra). D'altronde, lo stesso Torres Balbás aveva dedicato una qualche attenzione critica al castello di Vélez Blanco nell'articolo «De cómo desaparecen los antiguos palacios de la nobleza castellana» del 1923; v.:TORRES BALBÁS, Leopoldo. De cómo desaparecen los antiguos palacios de la nobleza castellana. In: Arquitectura. 1923, vol.V, n. ${ }^{\circ}$ 48, p. 105-109. Sulle zone amministrative negli anni Trenta v.: PÉREZ MARTÍN, José Luis Javier; RODRÍGUEZ SÁNCHEZ, Antonio; ARBAIZA BLANCO-SOLER, Silvia. Los arquitectos conservadores de zona. In: Anales de Edificación. 2017, vol, III, n. ${ }^{\circ} 3$, pp. $41-54$.

30 A questo primo incontro era presente anche Walter W. S. Cook, ringraziato dalla Raggio nel suo articolo dedicato al patio nel 1964; RAGGIO, Olga. The Vélez Blanco. Op. cit. (n. 1), p. 173. Sulla reja al Metropolitan:VINCENT, Clare. Precious Objects:Two Collections of European Smithing. In: The Metropolitan Museum of Art Bulletin. 1964, vol. XXII, n. ${ }^{\circ}$ 8, pp. 274-275. 
prima offerta avesse avuto un'eco negativa sullo staff del museo, avrebbe riferito di un diverso incontro con Nieto e col ministro de Educación Nacional, Jesús Rubio GarcíaMina, in allarme entrambi per una dura campagna di stampa attorno alle ultime dispersioni del patrimonio artistico nazionale; ragione per cui sembrava che la cessione dei frammenti di Vélez si fosse fatta più difficoltosa. A questa seconda sollecitazione, Rorimer avrebbe replicato, gelido:

«Dear Maria Luisa: [...] I received your letter.Yes, I did not feel that there was much I could say about the patio's cornice pieces [...]. I'm delighted to have heard your story even if it is a sad one»" ${ }^{31}$.

Una tanto estesa rete di messaggi e relazioni internazionali, intessuta dalla Raggio e favorita dai vertici del museo, rende del tutto implausibile la lettura delle successive vicende centrate attorno agli elementi architettonici rimasti a Vélez Blanco già avanzata da Miguel Guirao Gea all'inizio degli anni Sessanta ${ }^{32}$ e accolta, più di recente, da ulteriori riflessioni al proposito ${ }^{33}$ : lettura cioè che, nel migliore dei casi, attribuisce alla Raggio un ambizioso disegno di esproprio, giocato in una granitica opposizione con le autorità spagnole, locali e nazionali; e che nel peggiore le riferisce persino un intento proditorio nei confronti dei suoi interlocutori europei, fra l'Andalusia e Madrid.

Il coinvolgimento della nomenclatura franchista, già in questa prima fase della trasferta della Raggio, chiarisce infatti quanto la buona accoglienza rivolta alla studiosa dovesse rispecchiarsi in un'ampia strategia politica, in tutto coincidente con le contemporanee manovre governative indirizzate a un'efficace distensione dei rapporti con gli U.S.A.: nonostante il permanere nelle fasce conservatrici della società spagnola di un orgoglioso sentimento 'anti-yankee', è infatti difficile immaginare che la gestione della visita di una rappresentante del più prestigioso museo americano non venisse verificata sull'azione diplomatica allora condotta nei confronti di Washington e, in una prospettiva generale, dei cittadini statunitensi.

Del resto, il riattualizzarsi dell'offerta di acquisizione dei marmi, avanzata dal Met nel 1963, va interpretata sullo sfondo dei contatti avviati dalla Raggio negli anni precedenti; allo stesso tempo essa va inserita nel contesto delle nuove manovre intraprese quando la calendarizzazione del rimontaggio del patio al Met impose un andamento accellerato per l'intera pratica relativa alla struttura.

In vista di un simile impegno la Raggio si preoccupò infatti, fra la primavera del 1962

31 New York, Metropolitan Museum of Art, European Sculpture and Decorative Arts Department, Curatorial Files, 41.190.482: Blumenthal - installation-photos/notes/correspondence.

32 GUIRAO GEA, Miguel. Se va a empezar a restaurar el castillo de Vélez Blanco. Op. cit. (n. 20).

33 MOTOS DÍAZ, Ismael. La propuesta del Metropolitan Museum of Art de intercambio de elementos arquitectónicos del castillo de Vélez Blanco en 1963. In: Ph Investigación. 2014, n. ${ }^{\circ}$ 2, pp. 51-73 [ripubblicato in: Revista velezana. 2015, n. ${ }^{\circ} 33$, pp. 82-99]. 
e l'anno successivo, di stringere il dialogo con agli antichi padroni del castello, al fine di reperire la già menzionata maqueta della fortezza.Tali abboccamenti dovevano servirle per individuare anche inedite informazioni sulla storia della rocca, in particolare negli anni in cui l'avamposto passò fra i beni dei Fajardo. La studiosa stava infatti progettando di scrivere un articolo per il «Met Bulletin», da pubblicare in concomitanza con l'inaugurazione della nuova ala del museo, inclusiva del patio ricostituito. La sua indagine fu favorita, nel marzo 1962, da un passaggio a New York della marquesa de Martorell, Monica Müller Bravo, spintasi oltreoceano per il matrimonio di una figlia ${ }^{34}$. Grazie alla conoscenza stretta con la nobildonna, la Raggio fu infatti in grado di individuare il modello fra i beni della famiglia, coinvolta nel 1904 nella vendita dei marmi in quanto inclusa nell'eredità del XVIII duca di Medina Sidonia, José Joaquín Álvarez de Toledo (dal quale sarebbe loro derivata una comproprietà sulla fortezza andalusa) ${ }^{35}$. Dalla marchesa la studiosa ebbe anche notizia della presenza di documenti di rilievo proprio nell'archivio Medina Sidonia, allora in via di riordino nel palazzo familiare di Sanlúcar de Barrameda grazie agli sforzi della XXI duchessa, Luisa Isabel Álvarez de Toledo ${ }^{36}$.

Simultaneamente il Met riprese un'azione di networking tesa ad assicurarsi i frammenti superstiti. I precedenti spiegano bene il cambio di strategia, parzialmente perseguito in questo frangente. Se infatti si continuò nella ricerca di una trattativa diretta con $\mathrm{i}$ moderni proprietari della rocca $-\mathrm{e}$ cioè la famiglia del marqués de Valverde, Salvador Ferrandis y Álvarez de Toledo ${ }^{37}$ - si procedette anche per più ufficiali vie diplomatiche, coinvolgendo il console generale di Spagna a New York, Ángel Sanz-Briz.

La corrispondenza fra il museo, il rappresentante a Manhattan del governo spagnolo e le autorità di Madrid è già stata ripercorsa da Ismael Motos Díaz, basandosi sui documenti conservati presso l'Archivo

34 New York, Metropolitan Museum of Art, European Sculpture and Decorative Arts Department, Curatorial Files, 41.190.482: Blumenthal installation-photos/notes/correspondence: nota manoscritta di Raggio, senza data; memorandum di Raggio senza destinatario, 20 marzo 1962; lettera di Raggio alla marquesa de Martorell, 15 maggio 1962. Nel 1968, la Raggio doveva essersi fatta un'opinione diversa sull'importanza di questo modello; RAGGIO, Olga. El patio de Vélez Blanco. Op. cit. (n. 1), p. 234, n. 5.

35 Sulla vendita del patio: RUIZ GARCÍA, Alfonso. El castillo de Vélez Blanco (Almería).Vélez Rubio: Revista Velezana, Ayuntamiento de Vélez Rubio, 1999, pp. 105-109, 112-115; LENTISCO PUCHE, José Domingo (coord.). El castillo de Vélez Blanco. Op. cit. (n. 4), pp. 90-106; in ultimo: MOZZATI, Tommaso. Storia collezionistica. Op. cit. (n. 4), pp. 339-40; MOZZATI, Tommaso. The patio of Vélez Blanco. Op. cit. (n. 12), pp. 263-265.

36 New York, Metropolitan Museum of Art, European Sculpture and Decorative Arts Department, Curatorial Files, 41.190.482: Blumenthal installation-photos/notes/correspondence: memorandum di Raggio senza destinatario, 20 marzo 1962. Una lettera seguente, indirizzata alla Raggio dalla duquesa de Medina Sidonia, il 30 aprile 1963 (New York, Metropolitan Museum of Art, European Sculpture and Decorative Arts Department, Curatorial Files, 41.190.482: Blumenthal - installation-photos/notes/correspondence), invitava la studiosa a recarsi di persona a Sanlucár, al fine di condurre un'indagine estensiva nell'archivio. Questa visita non dovette però mai avvenire. In realtà, nella traduzione spagnola del suo articolo del '64 (approntata a quattro anni di distanza da Carmen Gómez Moreno su un testo in parte emendato), la stessa Raggio offriva una versione diversa della faccenda, ricordando come «a pesar de [...] muchos esfuerzos» non le fosse stato permesso di «explorar estos archivos»; RAGGIO, Olga. El patio de Vélez Blanco. Op. cit. (n. 1), p. 257, n. 48.

37 New York, Metropolitan Museum of Art, European Sculpture and Decorative Arts Department, Curatorial Files, 41.190.482: Blumenthal installation-photos/notes/correspondence: nota manoscritta di Raggio, senza data; lettera del marqués de Valverde a Raggio, 8 giugno 1965. 
General de la Administración: alle carte pubblicate in quell'articolo, si possono però aggiungere nuovi, importanti tasselli, provenienti dai files del Met. D'altronde quanto reso noto da Motos Díaz assume una più chiara evidenza se collocato sullo sfondo della vicenda fin qui raccontata, ignota allo studioso ${ }^{38}$.

Del tutto consapevoli dell'ostacolo istituzionale frapposto alla vendita dei marmi negli anni precedenti, Rorimer e la Raggio dovettero infatti avvertire l'esigenza di gestire il negoziato anche su un piano politico, cercando di riavviare la trattativa partendo da interlocutori più disponibili al dialogo.

Per questo, già il 25 gennaio 1963 Rorimer scrisse al console inviando il progetto di ripristino del patio approntato dalla studiosa, con l'intento di sensibilizzarlo sull'importanza di ottenere i frammenti mancanti per offrire una presentazione quanto più integra possibile del cortile e del suo arredo. A questa prima missiva, SanzBriz rispose il 7 febbraio, garantendo il suo intervento presso le autorità spagnole «about the six or seven fragments of the cornice of the patio of the castle». Al proposito, il console commentava lapidario: "we should do our best to avoid having to use modern substitutes». A ruota, sarebbe intervenuta la stessa Raggio, con un messaggio datato 15 febbraio, inteso per offrire al diplomatico un elenco completo dei marmi da recuperare nel pueblo andaluso. Nella nota dettagliata, la studiosa dichiarava inoltre: «It may be of interest to you to know that when I mentioned our need for these pieces of marble to the authorities in Vélez Blanco the alcalde, Don Miguel Ballesteros, and the Parish priest of the Church of Santiago- as well as to Don Francisco Prieto Moreno, I met with full understanding ${ }^{39}$.

È solo dopo aver preparato il terreno con questo interscambio che Rorimer arrivò a riprendere i contatti con Sanz-Briz nel marzo seguente (con la lettera nota a Motos Díaz), offrendo una permuta fra gli elementi presenti a Vélez Blanco e quelli, in possesso del Met, non inclusi nel piano di restituzione del cortile; azione evidentemente sollecitata

$38 \mathrm{Si}$ offre qui di seguito un elenco complessivo della documentazione presente nell'archivio del Metropolitan Museum of Art e nei files dell'European Sculpture and Decorative Arts Department (per la prima volta commentata nel corpo di questo articolo), integrante le carte rese note dall'articolo di Motos Díaz, e fondamentale per ricostruire il punto di vista del museo sulla vicenda. New York, Metropolitan Museum of Art, Archives, Office of the Secretary. Correspondence Files 1870-1950. Blumenthal George. Bequest - Patio (and Pipe Organ) 1952, 1954, 1963-64: lettera di Rorimer a Ángel Sanz-Briz, 13 marzo 1963; lettera di Sanz-Briz a Rorimer, 20 marzo 1963; lettera di Sanz-Briz a Alfonso de la Serna, 6 maggio 1963; lettera di De la Serna a Sanz-Briz, 13 maggio 1963; lettera di Sanz-Briz a Rorimer, 13 settembre 1963; lettera di Rorimer a Sanz-Briz, 18 settembre 1963. New York, Metropolitan Museum of Art, European Sculpture and Decorative Arts Department, Curatorial Files, 41.190.482: Blumenthal - installation-photos/ notes/correspondence): messaggio di Raggio a Rorimer, 16 gennaio 1959; lettera di Raggio a Prieto-Moreno, 2 settembre 1959; lettera di PrietoMoreno a Raggio, 15 settembre 1959; lettera di Raggio a Prieto-Moreno, 22 settembre 1959; lettera di Prieto-Moreno a Raggio, 23 novembre 1959; lettera di Raggio a Prieto-Moreno, 7 gennaio 1960; lettera di Prieto-Moreno a Raggio, 8 febbraio 1960; lettera di María Luisa Caturla a Rorimer, 5 luglio 1961; lettera di Caturla a Rorimer, 14 luglio 1961; lettera di Rorimer a Caturla, 17 luglio 1961; lettera di Rorimer a Sanz-Briz, 25 gennaio 1963; lettera di Sanz-Briz a Raggio, 8 febbraio 1963; lettera s.d. [timbro di ricezione: 8 febbraio 1963] di Sanz-Briz a Rorimer; lettera di Raggio a Sanz-Briz, 15 febbraio 1963.

39 Le due lettere citate in testo sono conservate in New York, Metropolitan Museum of Art, European Sculpture and Decorative Arts Department, Curatorial Files, 41.190.482: Blumenthal - installation photos/notes/correspondence. 
da quanto era stato suggerito nel '61 da Nieto Gallo, seppure nella prospettiva di un baratto più equo. Il Met infatti garantiva alla Spagna "two spandrels carved with grotesque", «an ensemble of three columns with doric capitals ... and two carved arches», illustrate da foto accluse al messaggio epistolare. Fra il marzo e il giugno le autorità madrilene formularono una risposta negativa, in un processo di valutazione che su spinta di Sanz-Briz coinvolse Alfonso de la Serna, director general de relaciones culturales del Ministerio de Asuntos Exteriores, lo stesso Nieto Gallo -il quale considerava la proposta «en principio aceptable»- oltre alla Real Academia de Bellas Artes de San Fernando. Proprio quest'ultima istituzione si assunse, di fatto, la responsabilità di un primo divieto, sulla base di una nota informativa composta da Diego Angulo Íñiguez per la Comisión Central de Monumentos. Il direttore delle Belle Arti scelse allora di interpellare la Real Academia de la Historia, la quale sottoscrisse il parere ostativo.Alla fine dell'estate la pratica venne dunque chiusa, con i ringraziamenti di rito: la lettera di Rorimer a Sanz-Briz del 18 settembre 1963 informava il diplomatico della volontà di non ricorrere a calchi dei pezzi mancanti, come offerto da Nieto Gallo in una lettera del 12 agosto, ma piuttosto di servirsi di integrazioni realizzate direttamente nel museo, a partire dagli elementi presenti nelle sue collezioni ${ }^{40}$.

La vicenda del riallestimento del patio divenne pertanto, dall'autunno '63, una 'storia americana'; contemporaneamente le autorità spagnole -su spinta dello stesso Prieto-Moreno- misero in cantiere un piano di salvaguardia del castello, arrivando a stilare un Proyecto de obras de consolidación y restauración della fortezza, attuatosi a partire dal $1964^{41}$.

La Raggio del resto, ancora prima dell'esito sfavorevole delle trattative, si diceva pienamente consapevole di quanto il programma di restituzione del cortile nell'edificio sulla Quinta dovesse essere interpretato in termini di compromesso. Non a caso, rientrata dall'Andalusia, aveva dichiarato in un messaggio confidenziale indirizzato a Rorimer l'8 giugno 1960: «our installation project would seem to be more correctly described as a re-adaptation of the Vélez architectural elements rather than as a strict architectural reconstruction. [...] Seen as a gallery, simply suggestive of a Spanish Renaissance Patio, the Museum's installation project appears as an harmonious and attractive area ${ }^{42}$.

Sulla base di questa e di altre analoghe riflessioni, ci si risolse pertanto a presentare

40 MOTOS DÍAZ, Ismael. La propuesta del Metropolitan Museum. Op. cit. (n. 33), pp. 59-64. Al proposito v. in particolare la corrispondenza fra Rorimer, la Raggio e il console Sanz-Briz; v. n. 38.

41 RUIZ GAR CÍA, Alfonso. El castillo de Vélez Blanco. Op. cit. (n. 35), pp. 115-6; LENTISCO PUCHE, José Domingo (coord.). El castillo de Vélez. Op. cit. (n. 4), pp. 174-200, 350-365; MOTOS DÍAZ, Ismael. La propuesta del Metropolitan Museum. Op. cit. (n. 33 ), p. 63.

42 New York, Metropolitan Museum of Art, European Sculpture and Decorative Arts Department, Curatorial Files, 41.190.482: Blumenthal installation-photos/notes/correspondence: report di Raggio a Rorimer, 8 giugno 1960. 
alla stampa la nuova sala del Met, alla sua inaugurazione nel novembre 1964, in termini coerenti con le premesse che ne avevano guidato il riassemblaggio. Non a caso, un divertito articolo a firma di John Canaday apparso sul popolare «The New York Times Magazine», istruiva i futuri visitatori dell'ala appena addizionata al museo, prevedendo come «to the money-minded it may appeal as a bit of picturesque old Spain transported to Fifth Avenue» mentre, di sicuro gli «avantgardists» si sarebbero risolti a razionalizzarne la struttura «as a prototype of the experiments in walk-in art called 'environments' ${ }^{43}$.

È del tutto conseguente allora che la Raggio -da sempre conscia dei limiti e dei pregi delle proposte formulate fra la fine degli anni Cinquanta e l'inizio degli anni Sessantatornasse a meditare sul lavoro condotto usando termini schiettamente celebrativi.

L'occasione le fu offerta da un simposio newyorkese, sponsorizzato assieme alla Direzione generale per la cooperazione culturale di Roma nel 1980, al quale la studiosa presentò un contributo -poco noto e ancor meno citato- il cui testo, sospeso fra memoir e revisione critica, intendeva far eco proprio agli entusiasmi immediati suscitati dalla messa in opera del patio.

Se infatti Rorimer, nel 1964, si era limitato a descrivere il cortile fra le glorie del museo ${ }^{44}$, la Raggio -nel riaffermare il proprio ruolo nella «single most important architectural installation carried out by the Metropolitan» attorno alla metà del secolo- attribuiva a quell'impresa una dimensione ulteriore: la studiosa cioè, pur nell'ammettere le molte mancanze del progetto, gli riconosceva, fra souvenirs commossi, la capacità di imporsi come un momento peculiare nella storia dell'istituzione, trasformando a un tempo le reliquie di Vélez Blanco in «a living part of our own artistic heritage». Il discorso dovette già allora apparire inequivoco: rivendicando il merito delle ricerche condotte con scrupolo a partire dal 1957-1958, la curatrice chiariva cioè come quel coscienzioso scandaglio avesse voluto non solo garantire al Metropolitan lo spettacolo magniloquente di un ulteriore capolavoro, ma avesse piuttosto inteso restituire al pubblico contemporaneo -immerso in un tempo dai chiari connotati politici- il prodotto straordinario di una storia artistica e culturale, capace per la sua stessa forza simbolica di parlare nuovamente al presente ${ }^{45}$.

43 CANADAY, John. Spanish Castle on Fifth Avenue. In: The New York Times Magazine. 22 novembre, 1964, pp. 106-107.

19

$\overline{\# 02 \quad 2021}$ 


\section{REFERENCIAS BIBLIOGRÁFICAS}

CANADAY, John. Spanish Castle on Fifth Avenue. In: The New York Times Magazine. 22 novembre, 1964, pp. 106-107.

FOX, Margalit. Olga Raggio, a Scholar and Art Curator, Dies at 82. In: The New York Times. 5 febbraio, 2009.

GALLEGO MORELL, Antonio. Antonio Gallego Burín (1895-1961). Madrid: Moneda y Crédito, 1973.

GALLEGO ROCA, Francisco Javier (ed.). Epistolario de Leopoldo Torres Balbás a Antonio Gallego Burín. Granada: Editorial Universidad de Granada, 1995.

GUIRAO GEA, Miguel. Se va a empezar a restaurar el castillo de Vélez Blanco. In: La voz de Almería. 19 agosto, 1964, p. 6.

HECKSCHER, Morrison H. The Metropolitan Museum of Art. An architectural History. In: The Metropolitan Museum of Art. Bulletin. 1995, vol. 53, n. ${ }^{\circ}$ 1, pp. 1-80.

https://doi.org/10.2307/3259465.

HIETIKKO, Brad. Los relieves artísticos del patio del Castillo deVélez Blanco en la casa de los Blumenthal (Nueva York) 1913. In: Revista velezana. 2017, n. ${ }^{\circ}$ 35, pp. 2-17.

HUSBAND, Timothy B. Creating the Cloisters. In: The Metropolitan Museum of Art Bulletin. 2013, vol. 70 , n. ${ }^{\circ} 4$.

LAMPÉREZY ROMEA,Vicente. Arquitectura civil española de los siglos I al XVIII. 2 vols. Madrid: Saturnino Calleja, 1922.

LENTISCO PUCHE, José Domingo (coord.). El castillo de Vélez Blanco 1506-2006. Imagen y Memoria. Almería: Centro de Estudios Velezanos, 2007.

LÓPEZ VEGA, Antonio. Gregorio Marañon. Radiografía de un liberal. Madrid:Taurus, 2011.

MARAÑÓN, Gregorio. Los tres Vélez. Una historia de todos los tiempos. Madrid: Espasa-Calpe, 1960.

MERINO DE CÁCERES, José Miguel; MARTÍNEZ RUIZ, María José. La destrucción del patrimonio artístico español.W. R. Hearst: el gran acaparador. Madrid: Cátedra, 2012.

MORADIELLOS, Enrique. The Potsdam Conference and the Spanish Question. In: Contemporary European History. 2001, vol. X, n. ${ }^{\circ}$ 1, pp. 73-90.

https://doi.org/10.1017/S0960777301001047.

MOTOS DÍAZ, Ismael. La propuesta del Metropolitan Museum of Art de intercambio de elementos arquitectónicos del castillo deVélez Blanco en 1963. In: Ph Investigación. 2014, n. ${ }^{\circ}$ 2, pp. $51-73$ [ripubblicato in: Revista velezana. 2015, n. ${ }^{\circ} 33$, pp. 82-99].

MOZZATI, Tommaso. The Vélez Blanco Patio and United States-Cuba Relationships in the 1950s. In: Metropolitan Museum Journal. 2021, n. 56.

MOZZATI, Tommaso. La venta del patio de Vélez Blanco en 1904. In: Revista velezana. 2020, n. ${ }^{\circ} 38$, pp. $2-11$.

MOZZATI, Tommaso. El patio de Vélez Blanco y la residencia de George Blumenthal en New York. In: Revista velezana. 2020, n. ${ }^{\circ}$ 38, pp. 13-23. https://doi.org/10.36901/allpanchis.v23i38.816. 
MOZZATI, Tommaso. Storia collezionistica del patio di Vélez Blanco: nuovi documenti e fotografie inedite. In: BSAA Arte. 2019, n. ${ }^{\circ} 85$, pp. 337-362. https://doi.org/10.24197/bsaaa.85.2019.337-362. MOZZATI, Tommaso. The patio of Vélez Blanco: a new Drawing and the Courtyard of the Fajardo Castle. In: Archivo español de arte. 2019, vol. XCII, n. ${ }^{\circ}$ 367, pp. 261-276.

MOSQUERA ADELL, Eduardo. Torres Balbás y Andalucía. In: RUIZ CABRERO, Gabriel; MUÑOZ COSME, Alfonso; SÁNCHEZ DEL BARRIO, Antonio (eds.). Leopoldo Torres Balbás y la restauración científica. Sevilla: Instituto Andaluz del Patrimonio Histórico-Patronato de la Alhambra y el Generalife, 2013, pp. 129-156.

MUÑOZ COSME, Alfonso. La vida y la obra de Leopoldo Torres Balbás. Sevilla: Instituto Andaluz de Patrimonio Histórico, 2005.

PÉREZ MARTÍN, José Luis Javier; RODRÍGUEZ SÁNCHEZ, Antonio; ARBAIZA BLANCOSOLER, Silvia. Los arquitectos conservadores de zona. In: Anales de Edificación. 2017, vol. III, n. ${ }^{\circ}$ 3, pp. 41-54. https://doi.org/10.20868/ade.2017.3677.

RAGGIO, Olga. The reconstruction of the Vélez Blanco Patio. In: The Preservation and Use of Artistic Cultural Heritage: Perspectives and Solutions. New York:The Metropolitan Museum of Art, 1980, pp. 120-126. RAGGIO, Olga. El patio de Vélez Blanco: un monumento señero del Renacimiento. GÓMEZMORENO, Carmen (trad.). In: Anales de la Universidad de Murcia Filosofía y Letras. 1968, vol. XXVI, n. ${ }^{\text {s }}$ 2-3, pp. 231-261.

RAGGIO, Olga. The Vélez Blanco patio: an Italian Renaissance Monument from Spain. In: The Metropolitan Museum of Art Bulletin. 1964, vol. 23, n. ${ }^{\circ}$ 4, pp. 142-176.

https://doi.org/10.2307/3258177.

ROMERO GALLARDO, Aroa. Prieto-Moreno, arquitecto conservador de La Alhambra (1936-1978). Razón y sentimiento. Granada: Editorial Universidad de Granada, 2014.

ROSENDORF, Neal M. Franco sells Spain to America. Hollywood, Tourism and Public Relations as Postwar Spanish Soft Power. New York: Palgrave MacMillan, 2014.

https://doi.org/10.1057/9781137372574.

RUIZ GARCÍA, Alfonso. El castillo de Vélez Blanco (Almería).Vélez Rubio: Revista Velezana, Ayuntamiento de Vélez Rubio, 1999.

TAPIA GARRIDO, José Ángel. Vélez Blanco, la villa señorial de los Fajardo. Vélez Blanco: Diputación Provincial de Almería, 1959.

TAYLOR, Francis Henry. The Blumenthal Collection. In: The Metropolitan Museum of Art Bulletin. 1941, vol. 36, n. ${ }^{\circ}$ 10, pp. 193, 195-198. https://doi.org/10.2307/3256519.

TORRES BALBÁS, Leopoldo. De cómo desaparecen los antiguos palacios de la nobleza castellana. In: Arquitectura. 1923, vol.V, n. ${ }^{\circ}$ 48, pp. 105-109.

VINCENT, Clare. Two Collections of European Smithing. In: The Metropolitan Museum of Art Bulletin. 1964, vol. XXII, n. ${ }^{\circ}$ 8, pp. 272-286.

VIÑES MILLET, Cristina. Antonio Gallego Burín. Granada: Comares, 2003. 
Figura 1. Patio di Vélez Blanco, ca. 1506/1509 - 1515, New York, The Metropolitan Museum of Art, inv. 41.190.482.

Figura 2. Mattie Edwards Hewitt, Casa Blumenthal, 1928, New York, The Metropolitan Museum of Art, Thomas J. Watson Library, Rare Books, coll. B1683771x, s. p. [ma fig. 10].

Figura 3. Autore sconosciuto, Casa Blumenthal, 1941, New York, The Metropolitan Museum of Art, Esda Department, Curatorial Files, 41.190.482.

Figura 4. Brown, Lawford and Forbes, Veduta meridionale della Thomas J. Watson Library, 1958. HECKSHER, M. H., The Metropolitan Museum of Art. An Architectural History, In: Metropolitan Museum of Art Bulletin, LIII, n. ${ }^{\circ}$ 1, estate 1995, p. 64.

Figura 5. J. Goldberg, Patio di Vélez Blanco, ca. 1904, acquerello, New York, The Hispanic Society of America, n. 144811.

Figura 6. Adolphe Giraudon, Frammento del patio di Vélez Blanco, ca. 1904-1905, New York, The Metropolitan Museum of Art, Esda Department, Curatorial Files, 41.190.482.

Figura 7. Olga Raggio, Castello di Vélez Blanco, 1959, New York, The Metropolitan Museum of Art, Esda Department, Curatorial Files, 41.190.482.

Figura 8. Francisco Prieto-Moreno, Castello di Vélez Blanco, 1959, New York, The Metropolitan Museum of Art, Esda Department, Curatorial Files, 41.190.482.

Figura 9. Olga Raggio, Castello di Vélez Blanco, 1959, New York, The Metropolitan Museum of Art, Esda Department, Curatorial Files, 41.190.482.

Figura 10. Francisco Prieto-Moreno, Castello di Vélez Blanco, 1959, New York, The Metropolitan Museum of Art, Esda Department, Curatorial Files, 41.190.482.

Figura 11. Olga Raggio, Castello di Vélez Blanco, 1959, New York, The Metropolitan Museum of Art, Esda Department, Curatorial Files, 41.190.482.

Figura 12. Leopoldo Torres Balbás, Castello di Vélez Blanco, ca. 1926, New York, The Metropolitan Museum of Art, Esda Department, Curatorial Files, 41.190.482. 


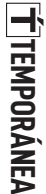

$\frac{23}{\# 02 \quad 2021}$ 



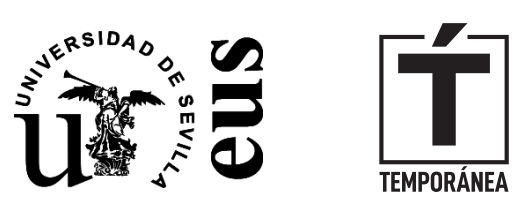

Editorial Universidad de Sevilla AÑO 2021.

e-ISSN 2659-8426. ISSN: 2695-7736

https://dx.doi.org/10.12795/TEMPORANEA 


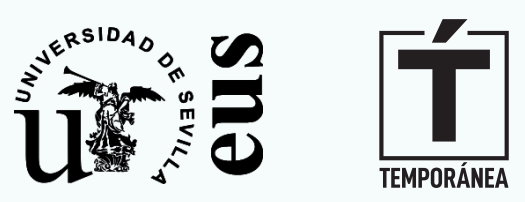

